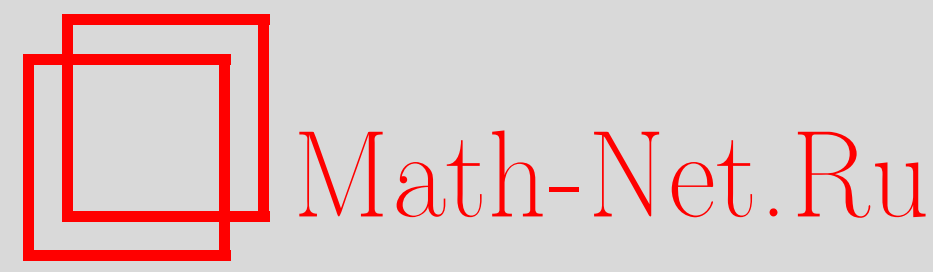

Ю. В. Кузьмин, Ю. С. Семенов, О гомологиях свободной нильпотентной группы класса 2, Матем. сб., 1998, том 189, номер 4, 49-82

DOI: https://doi.org/10.4213/sm308

Использование Общероссийского математического портала Math-Net.Ru подразумевает, что вы прочитали и согласны с пользовательским соглашением

http://www . mathnet.ru/rus/agreement

Параметры загрузки:

IP : 35.173 .137 .237

26 апреля 2023 г., $17: 38: 50$ 
УДК 512.54

\author{
Ю.В. Кузьмин, Ю.С. Семенов
}

\title{
О гомологиях свободной нильпотентной группы класса 2
}

Пусть $G$ - свободная нильпотентная группа класса 2 и пусть $\mathscr{G}$ - свободное нильпотентное кольо Ли класса 2 с тем же числом свободных образующих. Для группы $G$ строится свободная резольвента, которая как градуированный $\mathbb{Z} G$-модуль изоморфна $\mathbb{Z} G \otimes \Lambda(\mathscr{G})$, где $\mathbb{Z} G$ - групповое кольцо группш $G$, а $\Lambda(\mathscr{G})$ - внешняя алгебра кольца $\mathscr{G}$. Как следствие основной конструкции выводится изоморфизм целочисленных гомологий $H_{n} G \cong H_{n} \mathscr{G}$.

Библиография: 11 названий.

\section{Введение}

Пусть $G$ - конечно порожденная абелева группа. Хорошо известно, как строится достаточно удобная свободная резольвента тривиального $\mathbb{Z} G$-модуля $\mathbb{Z}$. Все сводится к циклическим группам, поскольку в качестве резольвенты для прямого произведения можно взять тензорное произведение резольвент сомножителей. Отсюда, например, легко следует, что если $G$ - свободная абелева группа, то ее внешняя алгебра над групповым кольцом $\mathbb{Z} G$, снабженная соответствующим дифференциалом, является резольвентой тривиального модуля $\mathbb{Z}$.

Картина резко меняется при переходе к нильпотентным группам. В общем случае кроме стандартной резольвенты ничего указать и нельзя. Причина понятна - нильпотентных групп слишком много и устроены они достаточно сложно. В такой ситуации естественно попытаться исследовать универсальный объект - общую точку задачи. В нашем случае - это свободная нильпотентная группа. Для разрешимых групп подобная точка зрения уже предлагалась в работах [1]-[4], где изучались, в частности, гомологии свободных разрешимых групп.

В этой статье мы изучаем гомологические свойства свободной нильпотентной группы класса 2. Основная идея состоит в использовании аналогии с кольцами Ли. Пусть $G$ - нильпотентная группа. Будем предполагать, что факторы нижнего центрального ряда группы $G$ - свободные абелевы группы. Как хорошо известно, на прямой сумме факторов нижнего центрального ряда можно ввести структуру градуированного кольца Ли, умножение в котором индуцируется коммутатором в группе. Обозначим это кольцо через $\mathscr{G}$. Учитывая тесную связь между группой $G$ и кольцом $\mathscr{G}$, естественно поставить вопрос о том, как связаны их гомологические свойства. Нетрудно, например, доказать совпадение гомологических размерностей: обе они равны сумме рангов факторов нижнего центрального ряда. Известно также [5], что изоморфны гомологии с рациональными коэффициентами.

Работа выполнена при финансовой поддержке Российского фонда фундаментальных исследований (грант № 97-01-00290). 
Если $G$ имеет класс 2 , то изоморфны даже гомологии с коэффициентами в кольце $\mathbb{Z}[1 /(d+1) !]$, где $d$ - ранг коммутанта [6]. Если $G$ - свободная нильпотентная группа класса 2 , то $\mathscr{G}$ - свободное нильпотентное кольцо класса 2 . Мы доказываем, что имеет место изоморфизм иелочисленных групп гомологий $H_{n} G \cong H_{n} \mathscr{G}$. Эта теорема получается как следствие основного результата - построения свободной резольвенты тривиального $\mathbb{Z} G$-модуля $\mathbb{Z}$, которая сходна с внешней алгеброй (отметим для сравнения и полноты картины, что в 1989 году И. Хюбшман предложил [7] некоторый общий способ построения резольвент для нильпотентных групп класса 2). Чтобы описать вкратце нашу конструкцию, начнем с кольца $\mathscr{G}$. Пусть $\Lambda=\Lambda(\mathscr{G})$ - внешняя алгебра аддитивной групшы кольца $\mathscr{G}$. На $\Lambda$ можно определить дифференциал $\partial$, который на внешнем произведении элементов $z_{1}, \ldots, z_{n} \in \mathscr{G}$ действует по формуле

$$
\partial\left(z_{1} \wedge \cdots \wedge z_{n}\right)=\sum_{i<j}(-1)^{i+j-1}\left[z_{i}, z_{j}\right] \wedge z_{1} \wedge \cdots \widehat{z}_{i} \cdots \widehat{z}_{j} \cdots \wedge z_{n}
$$

(как обычно “^” означает пропуск соответствующего элемента).

Мы строим некоторый подкомплекс в тривиализации стандартной резольвенты групшы $G$, который как градуированный $\mathbb{Z}$-модуль изоморфен алгебре $\Lambda$. Благодаря этому изоморфизму на $\Lambda$ возникает еше один дифференциал $d$. Он естественно распадается в сумму

$$
d=d_{2}+d_{3}+\cdots+d_{n}
$$

где $d_{2}=\partial$, а остальные слагаемые $d_{k}$ дают в некотором смысле все более точное приближение к дифференциалу группового комплекса. Если в соответствии c $(0.1) d_{2}$ получается выделением двух элементов $z_{i}$ и $z_{j}$ и добавлением коммутатора $\left[z_{i}, z_{j}\right]$, то $d_{k}$ определяется с помощью выделения $k$-элементных подмножеств и добавлением $k-1$ коммутатора. Мы доказьваем, что дифференциал $d$ можно поднять до некоторого дифференциала комплекса $\mathscr{R}(G)=\mathbb{Z} G \otimes \Lambda$, что превращает его в свободную резольвенту тривиального $\mathbb{Z} G$-модуля $\mathbb{Z}$. Это и есть наш основной результат. Затем мы строим цепное отображение комплекса $(\Lambda, d)$ в комплекс $(\Lambda, \partial)$, которое индуцирует изоморфизм групп гомологий, откуда следует, что $H_{n} G \cong H_{n} \mathscr{G}$.

$\mathrm{K}$ настоящему времени группы $H_{n} \mathscr{G}$ вычислены лишп для случая, когда число свободных образующих $r$ кольца $\mathscr{G}$ не превосходит 5 . Как оказалось, в группах $H_{n} \mathscr{G}$ имеется 3-кручение [8]. Неизвестно, появляются ли элементы других конечных порядков при $r>5$. С другой стороны, Шт. Зиггом [9] вычислены групшы рациональных гомологий $H_{n}(\mathscr{G}, \mathbb{Q})$.

Материал в статье расположен следующим образом. В первом параграфе мы даем точные формулировки основных результатов. Второй параграф̆ посвящен доказательству ряда утверждений (большей частью технических) о стандартном комплексе произвольной группы. Мы вводим понятие коммутатора двух цепей и доказываем формулу для дифференцирования перетасовочного произведения, которая на наш взгляд представляет самостоятельный интерес. В третьем параграфе строится подкомплекс $K(G)$ стандартного комплекса свободной нильпотентной групшы $G$ класса 2 , который затем (четвертый параграф)) поднимается до конечной свободной резольвенты. В пятом параграфе доказывается изоморфизм групп целочисленных гомологий групшы $G$ и соответствующего ей коль- 
ца Ли. В последнем параграфе доказано, что стабилизация спектральной последовательности Линдона-Хохшильда-Серра, ассоциированной с расширением $1 \rightarrow G^{\prime} \rightarrow G \rightarrow G / G^{\prime} \rightarrow 1$, происходит на третьем члене.

Авторы выражают признательность А. А. Клячко за полезные советы, касающиеся ориентации циклов.

\section{§1. Формулировка основных результатов}

1.1. Рассмотрим свободную нильпотентную группу $G=G(r)$ класса 2 с $r$ свободными образующими $x_{1}, \ldots, x_{r}$. Ее можно задать копредставлением

$$
\left.\left.G=\left\langle x_{i}, c_{i j}, 1 \leqslant i<j \leqslant r\right| c_{i j}=\left[x_{i}, x_{j}\right],\left[x_{k}, c_{i j}\right]=\left[c_{i j}, c_{k l}\right]=1\right]\right\rangle
$$

(групповой коммутатор $[a, b]$ равен $a b a^{-1} b^{-1}$ ).

Будем считать, что элементы $x_{1}, \ldots, x_{r}$ упорядочены в соответствии с естественным порядком индексов, т.е. $x_{1}<\cdots<x_{r}$, а коммутаторы $c_{i j}$ - лексикографически, т.е. $c_{i j}<c_{k l}$, если $j<l$ или $j=l, i<k$. Удобно также считать, что $c_{i j}<x_{k}$ для любых $i, j, k$. Пусть $\mathscr{G}$ - кольцо Ли, построенное по группе $G$, и $\Lambda(\mathscr{G})$ - его внешшяя алгебра над $\mathbb{Z}$. Поскольку $\Lambda(\mathscr{G})$ изоморфна внешней алгебре свободной абелевой групшы $G^{\prime} \oplus G / G^{\prime}$, то базу ее $n$-й однородной компоненты $\Lambda^{n}(\mathscr{G})$ образуют произведения вида

$$
c_{i_{1} j_{1}} \wedge \cdots \wedge c_{i_{q} j_{q}} \wedge x_{k_{1}} \wedge \cdots \wedge x_{k_{p}}, \quad p+q=n,
$$

в которых множители упорядочены по возрастанию (точнее, вместо свободных порождающих $x_{k}$ надо рассматривать их образы в $\left.G / G^{\prime}\right)$. Такие же произведения являются свободными образуюшими $\mathbb{Z} G$-модуля $\mathscr{R}(G)=\mathbb{Z} G \otimes \Lambda(\mathscr{G})$. Мы покажем, что на $\mathscr{R}(G)$ можно определить некоторый дифференциал $d$, причем если комплекс $(\mathscr{R}(G), d)$ пополнить в размерности 0 естественным эпиморфизмом $\mathbb{Z} G \rightarrow \mathbb{Z}$, то получится свободная резольвента тривиального модуля $\mathbb{Z}$.

Чтобы выписать явную формулу для дифференциала, введем некоторые дополнительные конструкции и обозначения.

1.2. Геометрическая интерпретация порождающих $x_{k}$ и коммутаторов $c_{i j}$. Пусть $\Delta=\Delta(r)$ - стандартный симплекс с $r$ вершинами, вложенный в $\mathbb{R}^{r}$ так, что $k$-й вершине соответствует точка $v_{k}(0, \ldots, 1,0, \ldots)$ (1 стоит на $k$-м месте). Зафиксируем ориентацию ребра $e_{i j}$, соединяюшего $v_{i}$ и $v_{j}$, считая его началом вершину с меньшим индексом. Геометрически это вектор $e_{i j}(0, \ldots,-1,0, \ldots, 1,0, \ldots)$. Удобно отождествить порождающие $x_{k}$ с вершинами $v_{k}$, а коммутаторы $c_{i j}, i<j$, с ориентированными ребрами $e_{i j}$. В частности, множества вершин и ребер становятся упорядоченньми. В дальнейшем указанное соответствие будет систематически использоваться.

1.3. Максимальные деревья и их четность. Пусть $T$ - максимальное дерево в 1-остове $\Delta^{1}(r)$ симплекса $\Delta(r)$. Очевидно, что $T$ имеет ровно $r$ вершин и $r-1$ ребро. Пусть $e_{1}(T), \ldots, e_{r-1}(T)$ - векторы, соответствуюшие ориентированньм ребрам дерева $T$, выписанным в порядке возрастания. Легко видеть, что они образуют базис пространства, состояшего из векторов с нулевой суммой координат. Если добавить к векторам $e_{i}(T)$ еше один вектор $e_{0}(1, \ldots, 1)$, то получится 
базис всего пространства $\mathbb{R}^{r}$. Пусть $\delta$ - определитель, составленньй из координат векторов $e_{0}, e_{1}(T), \ldots, e_{r-1}(T)$. Четностью $\varepsilon(T)$ дерева $T$ назовем 0 или 1 , если число $\delta$, соответственно, положительно или отрицательно. Таким образом, по определению $(-1)^{\varepsilon(T)}$ совпадает со знаком $\delta$.

Пусть теперь $V=\left\{v_{s_{1}}, \ldots, v_{s_{k}}\right\}$ - упорядоченное подмножество вершин симплекса $\Delta(r)$, т.е. $s_{1}<\cdots<s_{k}$. Рассмотрим грань $\Gamma(V) \subseteq \Delta(r)$, порожденную множеством $V$. Как симплициальные множества $\Gamma(V)$ и $\Delta(k)$ изоморфны: вершине $v_{s_{j}} \in \Gamma(V)$ соответствует вершина $v_{j} \in \Delta(k)$. Максимальному дереву $T \subseteq \Gamma^{1}(V)$ соответствует дерево $T^{\prime} \subseteq \Delta^{1}(k)$. По определению будем считать, что $\varepsilon(T)=\varepsilon\left(T^{\prime}\right)$. Условимся также, что $\max (V)$ обозначает множество всех максимальных деревьев $T \subseteq \Gamma^{1}(V)$.

1.4. Метка дерева. Пусть, как и ранњше, $T$ - максимальное дерево в $\Gamma^{1}(V)$. Добавление к $T$ еще одного ребра определяет в $\Gamma^{1}(V)$ некоторый цикл $Z$. Зафиксируем на нем ориентацию в соответствии с направлением, которое указывает старшее ребро (напомним, что все ребра в $\Gamma^{1}(V)$ ориентированы и упорядочены). Если $e$-произвольное ребро цикла $Z$, то оно также определяет на $Z$ некоторую ориентацию. Назовем ребро е положительным относительно $Z$, если эта ориентация совпадает с той, которую мы выбрали.

Определим теперь коммутаторную метку $и(T)$ дерева $T$. Пусть для каждого ребра $e c(e)$ - соответствующий ему коммутатор. Положим

$$
u(T)=\prod_{e} c(e)
$$

где произведение берется по всем ребрам $e \in \Gamma^{1}(V) \backslash T$, которые положительны относительно возникающих при их добавлении циклов.

ПримеР. Пусть $V=\left\{v_{1} \ldots v_{k}\right\}$ и $T$-звездное дерево с ребрами $e_{1}, i=2, \ldots, k$. Тогда все дополнительные ребра положительны относительно соответствующих циклов. Поэтому $u(T)=\prod_{1<i<j \leqslant k} c_{i j}$ - произведение коммутаторов, взятое по всем дополнительным ребрам. Напротив, если $T$ - простая цепь с ребрами $e_{i, i+1}$, $i=1, \ldots, k-1$, то ни одно дополнительное ребро не является положительным и $u(T)=1$.

1.5. Четность разбиения. Пусть $V$ - упорядоченное множество. Предположим, что $V$ представлено в виде дизъюнктного объединения своих подмножеств $V_{1}, \ldots, V_{h}$, которые к тому же расположены в фиксированном порядке. Такому разбиению соответствует перетасовка множества $V$, т.е. его перестановка, в которой сначала стоят элементы из $V_{1}$ в возрастающем порядке, затем элементы из $V_{2}$ и т. д. Четность этой перетасовки будем также называть четностью разбиения $V=V_{1} \sqcup \cdots \sqcup V_{h}$ и обозначать $\varepsilon\left(V_{1}, \ldots, V_{h}\right)$. В частности, любое подмножество $W \subseteq V$ определяет разбиение $V=W \sqcup(V \backslash W)$. Его четность будем для краткости обозначать просто $\varepsilon(W)$. Как обычно, $|W|$ будет обозначать число элементов множества $W$.

1.6. Сфрормулируем теперь основной результат. Будем использовать обозначения, введенные ранее. Кроме того, для любого множества вершин $V$ симплекса $\Delta(r)$ обозначим через $X(V)$ внешнее произведение элементов $x_{k}$, соответствующих вершинам $v_{k} \in V$, взятым в порядке возрастания. Аналогично, для любого 
множества $E$ ориентированных ребер обозначим через $C(E)$ упорядоченное внешнее произведение соответствуюших коммутаторов. В частности, для любого дерева $T \subseteq \Delta^{1}(r)$ определено произведение $C(T)$. Отметим, что в этих обозначениях образуюшие $(1.2) \mathbb{Z} G$-модуля $\mathscr{R}(G)$ запишутся в виде

$$
C(E) \wedge X(V), \quad E=\left\{e_{i_{1} j_{1}}, \ldots, e_{i_{q} j_{q}}\right\}, \quad V=\left\{v_{k_{1}}, \ldots, v_{k_{p}}\right\} .
$$

Пусть, наконец, для любого множества вершин $V x(V)$ - инверсное произведение элементов $x_{k}$, соответствуюших вершинам $v_{k} \in V$, взятым в порядке убывания. Подчеркнем, что речь идет об умножении в групте, т.е. $x(V) \in G$.

Tеорема 1. Пусть $\mathbb{Z} G$-гомоморфизм $d: \mathscr{R}(G) \rightarrow \mathscr{R}(G)$ задан на свободных образующих (1.3) формулой

$$
\begin{aligned}
& d(C(E) \wedge X(V))=-\sum_{s=1}^{q}(-1)^{s-1}\left(c_{i_{s} j_{s}}-1\right) \otimes C\left(E \backslash e_{i_{s} j_{s}}\right) \wedge X(V) \\
& +\sum_{t=1}^{p}(-1)^{q+t-1}\left(x_{k_{t}}-1\right) \otimes C(E) \wedge X\left(V \backslash v_{k_{t}}\right) \\
& \quad+\sum_{\substack{W \subseteq V,|W| \geqslant 2 \\
T \in \max (W)}}(-1)^{q+\varepsilon(W)+\varepsilon(T)} x(W) u(T) \otimes C(E) \wedge C(T) \wedge X(V \backslash W),
\end{aligned}
$$

где в последней группе слагаемых суммирование происходит по всем подмножествам $W \subseteq V$, содержащим не менее двух әлементов, и по всем максимальным деревьям $T \in \max (W)$. Тогда $d^{2}=0$ и соответствующий пополненный комплекс $\mathscr{R}(G) \rightarrow \mathbb{Z}$ с дифференциалом $d$ является свободной резольвентой тривиального $\mathbb{Z} G$-модуля $\mathbb{Z}$.

Отметим, что эта резольвента является минимальной в том смысле, что ее длина равна гомологической размерности $h$ групшы $G$, т.е. равна $r+\left(\begin{array}{c}r \\ 2\end{array}\right)=\left(\begin{array}{c}r+1 \\ 2\end{array}\right)$. Число свободных образующих однородной компоненты степени $n$ равно $\left(\begin{array}{l}h \\ n\end{array}\right)$. Домножая резольвенту $\mathscr{R}(G)$ тензорно на $\mathbb{Z}$ над $\mathbb{Z} G$, мы получим комплекс $\Lambda(\mathscr{G})$, дифференциал которого также обозначим через $d$. В $\Lambda(\mathscr{G})$

$$
d(C(E) \wedge X(V))=\sum_{\substack{W \subseteq V,|W| \geqslant 2 \\ T \in \max (W)}}(-1)^{\mu} C(E) \wedge C(T) \wedge X(V \backslash W),
$$

где $\mu=|E|+\varepsilon(W)+\varepsilon(T)$. Естественно представить $d$ в виде

$$
d=d_{2}+d_{3}+\cdots+d_{r},
$$

где $d_{2}(C(E) \wedge X(V))$ - это те слагаемые в выписанной сумме, которые соответствуют 2-элементным подмножествам $W, d_{3}$ - те, которые соответствуют 3 -элементньм подмножествам, и т. д. Для $d_{2}$ получается следуюшее выражение:

$$
\begin{aligned}
d_{2}(C(E) & \left.\wedge x_{k_{1}} \wedge \cdots \wedge x_{k_{p}}\right) \\
= & \sum_{i<j}(-1)^{|E|+i+j-1} C(E) \wedge\left[x_{k_{i}}, x_{k_{j}}\right] \wedge x_{k_{1}} \wedge \cdots \widehat{x}_{k_{i}} \cdots \widehat{x}_{k_{j}} \cdots \wedge x_{k_{p}},
\end{aligned}
$$

что совпадает с дифференциалом $\partial$ комплекса $(\Lambda(\mathscr{G}), \partial)$, с помощью которого вычисляются целочисленные гомологии кольца $\mathscr{G}$. Оказывается, что несмотря на дополнительные слагаемые $d_{k}, k>2$, комплексы $(\Lambda(\mathscr{G}), d)$ и $(\Lambda(\mathscr{G}), \partial)$ изоморфны. 
ТЕОРема 2. Существует автоморфизм $F$ градуированного $\mathbb{Z}$-модуля $\Lambda(\mathscr{G})$, являющийся цепным отображением комплекса $(\Lambda(\mathscr{G}), \partial)$ в комплекс $(\Lambda(\mathscr{G}), d)$.

Отсюда в качестве следствия получается

ТЕОРема 3. Для любого $n \geqslant 0$ справедливо следующее: $H_{n} G \cong H_{n} \mathscr{G}$.

Разумеется изоморфны и группы целочисленных когомологий, так как по формуле универсальных коэффициентов они выражаются через гомологии.

Рассмотрим расширение

$$
1 \rightarrow G^{\prime} \rightarrow G \rightarrow G / G^{\prime} \rightarrow 1
$$

По нему можно построить спектральную последовательность Линдона-Хохшильда-Серра, сходяшуюся к целочисленным гомологиям группы $G$

$$
E_{p q}^{2}=H_{p}\left(G / G^{\prime}\right) \otimes H_{q}\left(G^{\prime}\right) \cong \Lambda^{p}\left(G / G^{\prime}\right) \otimes \Lambda^{q}\left(G^{\prime}\right) .
$$

Дифференциал $d_{p q}^{2}$ совпадает с умножением на 2-коцикл, задаюший расширение (1.4), откуда можно вывести, что

$$
\begin{aligned}
& d_{p q}^{2}\left(x_{k_{1}} \wedge \cdots \wedge x_{k_{p}} \otimes c_{i_{1} j_{1}} \wedge \cdots \wedge c_{i_{q} j_{q}}\right) \\
& \quad=\sum_{i<j}(-1)^{i+j-1}\left[x_{k_{i}}, x_{k_{j}}\right] \wedge x_{k_{1}} \wedge \cdots \widehat{x}_{k_{i}} \cdots \widehat{x}_{k_{j}} \cdots \wedge x_{k_{p}} \otimes c_{i_{1} j_{1}} \wedge \cdots \wedge c_{i_{q} j_{q}} .
\end{aligned}
$$

Это означает, что $d_{p q}^{2}$ совпадает с дифференциалом комплекса, с помощью которого вычисляются группы $H_{n} \mathscr{G}$. Из этого наблюдения и теоремы 3 мы выводим следуюший результат.

Теорема 4. В спектральной последовательности расширения (1.4) дифференциаль $d_{p q}^{3}$ тривиальны, т.е. спектральная последовательность стабилизируется в члене $E^{3}$. Более того, соответствующая фильтрация на $H_{n} G$ расщепляется и $H_{n} G \cong \bigoplus_{p+q=n} E_{p q}^{3}$.

\section{§2. Коммутаторы в стандартном групповом комплексе}

2.1. Рассмотрим произвольную группу $G$. Стандартный (нормализованный) комплекс $C(G)$ определяется следуюшим образом: при $n \geqslant 1$ группа $n$-мерных цепей $C_{n}(G)$ - это свободный $\mathbb{Z}$-модуль с базисом, состояшим из всевозможных строк $\left(g_{1}, g_{2}, \ldots, g_{n}\right)$, где $g_{i} \in G \backslash\{1\} ; C_{0}(G)$ порождается пустой строкой ( ), т.е. $C_{0}(G) \cong \mathbb{Z}$.

Дифференциал $d: C_{n}(G) \rightarrow C_{n-1}(G)$ действует на базисных элементах по правилу

$d\left(g_{1}, \ldots, g_{n}\right)=\left(g_{2}, \ldots, g_{n}\right)+\sum_{i=1}^{n}(-1)^{i}\left(g_{1}, \ldots, g_{i} g_{i+1}, \ldots, g_{n}\right)+(-1)^{n}\left(g_{1}, \ldots, g_{n-1}\right)$.

Группы гомологий комплекса

$$
\cdots \stackrel{d}{\longrightarrow} C_{n}(G) \stackrel{d}{\longrightarrow} C_{n-1}(G) \stackrel{d}{\longrightarrow} \cdots
$$


изоморфны группам гомологий $H_{n}(G, \mathbb{Z})=H_{n} G$.

Хорошо известно, что градуированный модуль $C(G)=\bigoplus_{n \geqslant 0} C_{n}(G)$ можно превратить в градуированную комму тативную ассоциативную алгебру с единицей с помощью перетасовочного умножения. Напомним, как оно определяется. Пусть $x=\left(x_{1}, \ldots, x_{k}\right), \quad y=\left(y_{1}, \ldots, y_{m}\right)$. Рассмотрим упорядоченное множество индексов $N=\{1, \ldots, k+m\}$. Если $J=\left\{i_{1}, \ldots, i_{k}\right\}$ - подмножество мощности $k$ в $N$, то $J$-перетасовка - это строка

$$
(x, y)_{J}=\left(y_{1}, \ldots, x_{1}, \ldots, x_{k}, \ldots, y_{m}\right)
$$

$\left(x_{p}\right.$ стоит на $i_{p}$-м месте, порядок $x_{i}$ и $y_{j}$ по отдельности такой же, как в исходных строках). Обозначим $\widetilde{J}=\sum_{p=1}^{k}\left(i_{p}-p\right)$ - четность перетасовки $(x, y)_{J}$. Тогда перетасовочное произведение $x$ и $y$ определяется равенством

$$
x * y=\sum_{J}(-1)^{\widetilde{J}}(x, y)_{J}
$$

в котором $J$ пробегает все подмножества мощности $k$ в $N$.

По линейности перетасовочное произведение можно распространить на весь комплекс $C(G)$. Например, для любых $g_{i} \in G$

$$
\left(g_{1}\right) *\left(g_{2}\right) * \cdots *\left(g_{k}\right)=\sum_{\pi \in S_{k}}(-1)^{\widetilde{\pi}}\left(g_{\pi(1)}, \ldots, g_{\pi(k)}\right),
$$

где $\pi$ пробегает симметрическую группу $S_{k}, \widetilde{\pi}$ - четность $\pi$.

2.2. Умножение и дифференциал в $C(G)$. Для однородного элемента $u \in$ $C_{n}(G)$ положим $\widetilde{u}=n$.

ОПРЕДЕЛЕНИЕ 2.1. Коммутатором әлементов $x \in C_{k}(G), y \in C_{m}(G)$ называется элемент $[x, y] \in C_{k+m-1}(G)$ такой, что

$$
[x, y]=d(x * y)-d x * y-(-1)^{\widetilde{x}} x * d y .
$$

Другими словами, $[x, y]$ - это поправка к "формуле Лейбница" в алгебре $C(G)$ с дифференциалом $d$.

Чтобы получить явную формулу для коммутатора, введем следующие обозначения. Пусть $x=\left(x_{1}, \ldots, x_{k}\right) \in C_{k}(G)$. Для любого $j, 1 \leqslant j \leqslant k-1$, положим $L_{j}(x)=\left(x_{1}, \ldots, x_{j}\right), R_{j}(x)=\left(x_{j+1}, \ldots, x_{k}\right)$. Кроме того, по определению $L_{0}(x)=()=R_{k}(x), L_{k}(x)=x=R_{0}(x)$. Для двух строк $x=\left(x_{1}, \ldots, x_{k}\right)$ и $y=\left(y_{1}, \ldots, y_{m}\right)$ определим операцию конкатенации (приписывания): $x \circ y=$ $\left(x_{1}, \ldots, x_{k}, y_{1}, \ldots, y_{m}\right)$. По линейности конкатенация продолжается на $C(G)$. Отметим, что $x=L_{j}(x) \circ R_{j}(x)$.

Лемма 2.1. Пусть $x=\left(x_{1}, \ldots, x_{k}\right)$ u $y=\left(y_{1}, \ldots, y_{m}\right)$. Тогда $[x, y]$ имеет вид:

$$
\sum_{p=1}^{k} \sum_{q=1}^{m}(-1)^{p+q(k-p)}\left(L_{p-1}(x) * L_{q-1}(y)\right) \circ\left(\left(x_{p} y_{q}\right)-\left(y_{q} x_{p}\right)\right) \circ\left(R_{p}(x) * R_{q}(y)\right) .
$$


ДокаЗАтельство. Рассмотрим $d(x * y)=\sum_{J}(-1)^{\widetilde{J}} d(x, y)_{J}$. Дифференциал произвольной перетасовки

$$
d(x, y)_{J}=d\left(y_{1}, \ldots, x_{1}, \ldots, x_{k}, \ldots, y_{m}\right),
$$

где $J=\left\{i_{1}, \ldots, i_{k}\right\}$, равен сумме слагаемых, каждое из которых может быть отнесено к одному из следующих четырех типов.

1) Тип $(x x)$. Сюда относятся слагаемые вида $(-1)^{t}\left(y_{1}, \ldots, x_{p} x_{p+1}, \ldots, y_{m}\right)$, а также слагаемые, в которых отсутствуют либо $x_{1}$, либо $x_{k}$.

2) Тип (уу). Сюда относятся слагаемые вида $(-1)^{s}\left(y_{1}, \ldots, y_{q} y_{q+1}, \cdots, y_{m}\right)$, а также слагаемые, в которых отсутствует либо $y_{1}$, либо $y_{m}$.

3) Тип $(x y)$. К нему относятся слагаемые вида $(-1)^{a}\left(y_{1}, \ldots, x_{p} y_{q}, \ldots, y_{m}\right)$.

4) Тип $(y x)$. K нему относятся слагаемые вида $(-1)^{b}\left(y_{1}, \ldots, y_{q} x_{p}, \ldots, y_{m}\right)$.

Покажем, что сумма всех слагаемых типа $(x x)$ равна $d x * y$. Рассмотрим, например,

$$
(-1)^{\widetilde{J}}\left(y_{1}, \ldots, x_{p}, x_{p+1}, \ldots, y_{m}\right) .
$$

В данном случае $J=\left\{i_{1}, \ldots, i_{p}, i_{p}+1, \ldots, i_{k}\right\}$. Дифференцирование дает слагаемое $(-1)^{t}\left(y_{1}, \ldots, x_{p} x_{p+1}, \ldots, y_{m}\right)$, где

$$
t=i_{p}+\sum_{j=1}^{k}\left(i_{j}-j\right) \equiv p+\sum_{j=1}^{p}\left(i_{j}-j\right)+\sum_{j=p+2}^{k}\left(i_{j}-j\right) \quad(\bmod 2) .
$$

$\mathrm{C}$ другой стороны, в $d x * y$ имеется соответствуюшее слагаемое

$$
\begin{aligned}
& (-1)^{s}\left(y_{1}, \ldots, x_{p} x_{p+1}, \ldots, y_{m}\right), \text { где } \\
& s=p+\sum_{j=1}^{p}\left(i_{j}-j\right)+\sum_{j=p+2}^{k}\left(i_{j}-1-(j-1)\right) \equiv t \quad(\bmod 2) .
\end{aligned}
$$

Аналогично рассматриваются слагаемые типа $(x x)$, в которых отсутствует либо $x_{1}$, либо $x_{k}$. Точно так же все слагаемые типа (yy) в сумме дают $(-1)^{\widetilde{x}} x * d y$. Отсюда можно сделать вывод, что $[x, y]$ в точности равен сумме всех слагаемых типа $(x y)$ и $(y x)$.

Исследуем слагаемое типа $(x y)$

$$
(-1)^{a}\left(y_{1}, \ldots, x_{p} y_{q} \ldots, y_{m}\right)
$$

при фиксированных $p, q$. Очевидно, что $x_{p} y_{q}$ стоит на $(p+q-1)$-м месте в строке; начало строки есть некоторая перетасовка $L_{p-1}(x)$ и $L_{q-1}(y)$, конец строки также есть некоторая перетасовка $R_{p}(x)$ и $R_{q}(y)$. Таким образом, можно записать, что

$$
\begin{aligned}
& (-1)^{a}\left(y_{1}, \ldots, x_{p} y_{q} \ldots, y_{m}\right) \\
& \quad=(-1)^{a}\left(L_{p-1}(x), L_{q-1}(y)\right)_{J_{1}} \circ\left(x_{p} y_{q}\right) \circ\left(R_{p}(x), R_{q-1}(y)\right)_{J_{2}},
\end{aligned}
$$

где

$$
\begin{aligned}
& J_{1}=\left\{i_{1}, \ldots, i_{p-1}\right\} \subset\{1, \ldots, p+q-2\}, \\
& J_{2}=\left\{i_{p+1}, \ldots, i_{k}\right\} \subset\{p+q+1, \ldots, k+m\},
\end{aligned}
$$


причем

$$
\begin{aligned}
a & =p+q-1+\sum_{j=1}^{p-1}\left(i_{j}-j\right)+p+q-1-p+\sum_{j=p+1}^{k}\left(i_{j}-j\right) \\
& =p+\widetilde{J}_{1}+\sum_{j=p+1}^{k}\left(i_{j}-j-q\right)+q(p-k)=p+q(k-p)+\widetilde{J}_{1}+\widetilde{J}_{2} .
\end{aligned}
$$

Отсюда получаем, что сумма всех слагаемых вида

$$
(-1)^{a}\left(y_{1}, \ldots, x_{p} y_{q}, \ldots, y_{m}\right)
$$

при фиксированных $p, q$ (они соответствуют всевозможным парам $J_{1}, J_{2}$ ) равна

$$
\begin{aligned}
\sum_{J_{1}, J_{2}}(-1)^{p+q(k-p)+\widetilde{J}_{1}+\widetilde{J}_{2}}\left(L_{p-1}(x), L_{q-1}(y)\right)_{J_{1}} \circ\left(x_{p} y_{q}\right) \circ\left(R_{p}(x), R_{q}(y)\right)_{J_{2}} \\
=(-1)^{p+q(k-p)}\left(L_{p-1}(x) * L_{q-1}(y)\right) \circ\left(x_{p} y_{q}\right) \circ\left(R_{p}(x) * R_{q}(y)\right) .
\end{aligned}
$$

Аналогично рассматриваются слагаемые типа $(y x)$. Суммируя теперь по всем $p$ от 1 до $k$ и по всем $q$ от 1 до $m$, получим утверждение леммы.

Отметим очевидные следствия леммы 2.1.

СЛЕДСТВИЕ 2.1. Если все $x_{i}$ из $\left(x_{1}, \ldots, x_{k}\right)$ коммутируют со всеми $y_{j}$ из $\left(y_{1}, \ldots, y_{m}\right), m o$

$$
[x, y]=0, \quad d(x * y)=d x * y+(-1)^{\widetilde{x}} x * d y
$$

СлЕДСТВИЕ 2.2. Пусть с лежит в центре группь $G$. Тогда для любого $y \in C(G)$ справедливо следующее: $d((c) * y)=-(c) * d y$.

В дальнейших параграфах появятся элементы комплекса $C(G)$ специального вида, а именно, конкатенации $(g) \circ\left(\left(c_{1}\right) * \cdots *\left(c_{k}\right)\right), g, c_{i} \in G$. Такие элементы мы для краткости будем обозначать $\left(g ; c_{1} * \cdots * c_{k}\right)$. Отметим, что

$$
\left(g ; c_{1} * \cdots * c_{k}\right)=\sum_{\pi \in S_{k}}(-1)^{\widetilde{\pi}}\left(g, c_{\pi(1)}, \ldots, c_{\pi(k)}\right) .
$$

Следующие две леммы описывают свойства этих элементов.

ЛЕмма 2.2. Если все $c_{i}, i=1, \ldots, k+m$, попарно коммутируют и коммутируют с $g, h \in G$, mo

$$
\begin{aligned}
& {\left[\left(g ; c_{1} * \cdots * c_{k}\right),\right.}\left.\left(h ; c_{k+1} * \cdots * c_{k+m}\right)\right] \\
&=(-1)^{k+1}\left(\left(g h ; c_{1} * \cdots * c_{k+m}\right)-\left(h g ; c_{1} * \cdots * c_{k+m}\right)\right)
\end{aligned}
$$


ДоказАТеЛЬство. Пусть $\pi \in S_{k}, \sigma \in S_{m}$ - некоторые перестановки. По лемме 2.1 и условию леммы 2.2

$$
\begin{aligned}
& (-1)^{\tilde{\pi}+\widetilde{\sigma}}\left[\left(g, c_{\pi(1)}, \ldots, c_{\pi(k)}\right),\left(h, c_{k+\sigma(1)}, \ldots, c_{k+\sigma(m)}\right)\right] \\
& \quad=(-1)^{k+1+\widetilde{\pi}+\widetilde{\sigma}}((g h)-(h g)) \circ\left(\left(c_{\pi(1)}, \ldots, c_{\pi(k)}\right) *\left(c_{k+\sigma(1)}, \ldots, c_{k+\sigma(m)}\right)\right) .
\end{aligned}
$$

Суммируя по всем $\pi \in S_{k}, \sigma \in S_{m}$, получаем утверждение леммы.

Лемма 2.3. Пусть все $c_{i} \in G$ попарно коммутируют. Тогда

$$
\begin{aligned}
& d\left(g ; c_{1} * \cdots * c_{k}\right)=\left(c_{1}\right) * \cdots *\left(c_{k}\right) \\
& \quad+\sum_{p=1}^{k}(-1)^{p-1}\left(\left(g ; c_{1} * \cdots \widehat{c_{p}} \cdots * c_{k}\right)-\left(g c_{p} ; c_{1} * \cdots \widehat{c_{p}} \cdots * c_{k}\right)\right) .
\end{aligned}
$$

ДокАЗАТЕльство. Справедлива следующая цепочка равенств:

$$
\begin{aligned}
d\left(g ; c_{1} * \cdots * c_{k}\right)= & \sum_{\pi \in S_{k}}(-1)^{\tilde{\pi}} d\left(g ; c_{\pi(1)}, \ldots, c_{\pi(k)}\right) \\
= & \sum_{\pi \in S_{k}}(-1)^{\tilde{\pi}}\left(c_{\pi(1)}, \ldots, c_{\pi(k)}\right) \\
& -\sum_{\pi \in S_{k}}(-1)^{\widetilde{\pi}}\left(g c_{\pi(1)}, c_{\pi(2)}, \ldots, c_{\pi(k)}\right) \\
& +(-1)^{k+1} \sum_{\pi \in S_{k}}(-1)^{\widetilde{\pi}}\left(g, c_{\pi(1)}, \ldots, c_{\pi(k-1)}\right)
\end{aligned}
$$

(все остальные слагаемые взаимно уничтожаются ввиду попарной перестановочности $c_{i}$ в $\left.G\right)$. При этом

$$
\begin{aligned}
& \sum_{\pi \in S_{k}}(-1)^{\widetilde{\pi}}\left(c_{\pi(1)}, \ldots, c_{\pi(k)}\right)=\left(c_{1}\right) * \cdots *\left(c_{k}\right), \\
& \sum_{\pi \in S_{k}}(-1)^{\widetilde{\pi}}\left(g c_{\pi(1)}, c_{\pi(2)}, \ldots, c_{\pi(k)}\right)=\sum_{p=1}^{k} \sum_{\pi: \pi(1)=p}(-1)^{\widetilde{\pi}}\left(g c_{p}, c_{\pi(2)}, \ldots, c_{\pi(k)}\right) \\
& =\sum_{p=1}^{k}(-1)^{p-1}\left(g c_{p} ; c_{1} * \cdots \widehat{c_{p}} \cdots * c_{k}\right) \text {, } \\
& \sum_{\pi \in S_{k}}(-1)^{\tilde{\pi}+k+1}\left(g, \ldots, c_{\pi(k-1)}\right)=\sum_{p=1}^{k} \sum_{\pi: \pi(k)=p}(-1)^{\tilde{\pi}+k+1}\left(g, \ldots, c_{\pi(k-1)}\right) \\
& =\sum_{p=1}^{k}(-1)^{p-1}\left(g ; c_{1} * \cdots \widehat{c_{p}} \cdots * c_{k}\right) \text {. }
\end{aligned}
$$

Тем самым лемма доказана. 
2.3. Формула дифференцирования. Начнем со вспомогательных лемм. Пусть $x=\left(x_{1}, \ldots, x_{k}\right) \in C_{k}(G), y=\left(y_{1}, \ldots, y_{m}\right) \in C_{m}(G)$. Выберем произвольные $u=\left(u_{1}, \ldots, u_{t}\right), v=\left(v_{1}, \ldots, v_{q}\right),(f) \in C_{1}(G)$, зафиксируем некоторые числа $s$ от 1 до $k, n$ от 0 до $m$ и рассмотрим всевозможные подмножества $J=\left\{i_{1}, \ldots, i_{k}\right\} \subset\{1, \ldots, k+m\}$ такие, что $i_{s}=s+n$.

Лемма 2.4. Имеет место формула:

$$
\begin{aligned}
& \sum_{J: i_{s}=s+n}(-1)^{\widetilde{J}}\left(u * L_{s+n-1}\left((x, y)_{J}\right)\right) \circ(f) \circ\left(v * R_{s+n}\left((x, y)_{J}\right)\right) \\
& \quad=(-1)^{n(k-s+1)}\left(u * L_{s-1}(x) * L_{n}(y)\right) \circ(f) \circ\left(v * R_{s}(x) * L_{n}(y)\right) .
\end{aligned}
$$

ДокаЗАТЕЛЬСтво. Если $i_{s}=s+n$ фиксировано, то $x_{s}$ стоит на $(s+n)$-м месте и $L_{s+n-1}\left((x, y)_{J}\right)$ есть некоторая перетасовка $\left(L_{s-1}(x), L_{n}(y)\right)_{J_{1}}$, где $J_{1}=\left\{i_{1}, \ldots, i_{s-1}\right\} \subset\{1, \ldots, s+n-1\} . \quad$ Аналогично, $R_{s+n}\left((x, y)_{J}\right)$ есть $\left(R_{s}(x), R_{n}(y)\right)_{J_{2}}$, где $J_{2}=\left\{i_{s+1}, \ldots, i_{k}\right\} \subset\{s+n+1, \ldots, k+m\}$. При этом

$$
\begin{aligned}
\widetilde{J} & =\sum_{p=1}^{k}\left(i_{p}-p\right)=\sum_{p=1}^{s-1}\left(i_{p}-p\right)+s+n-s+\sum_{p=s+1}^{k}\left(i_{p}-p\right) \\
& =n+\widetilde{J}_{1}+\sum_{p=s+1}^{k}\left(i_{p}-p-n\right)+n(k-s)=n(k-s+1)+\widetilde{J}_{1}+\widetilde{J}_{2} .
\end{aligned}
$$

Таким образом, левая часть (2.1) равна

$$
\begin{aligned}
\sum_{J_{1}, J_{2}}(-1)^{\widetilde{J}}(u & \left.*\left(L_{s-1}(x), L_{n}(y)\right)_{J_{1}}\right) \circ(f) \circ\left(v *\left(R_{s}(x), R_{n}(y)\right)_{J_{2}}\right) \\
& =(-1)^{n(k-s+1)}\left(u * L_{s-1}(x) * L_{n}(y)\right) \circ(f) \circ\left(v * R_{s}(x) * R_{n}(y)\right),
\end{aligned}
$$

что и требовалось доказать.

Лемма 2.5. Пусть $b=\left(b_{1}, \ldots, b_{t}\right) \in C_{t}(G), z=\left(z_{1}, \ldots, z_{q}\right) \in C_{q}(G)$. Тогда

$$
b * z=\sum_{n=0}^{q}(-1)^{n(t-j+1)}\left(L_{j-1}(b) * L_{n}(z)\right) \circ\left(b_{j}\right) \circ\left(R_{j}(b) * R_{n}(y)\right)
$$

для любого $\mathrm{j}$ от 1 до $t$.

ДокаЗАТЕЛЬСТво. Пусть $J=\left\{i_{1}, \ldots, i_{t}\right\} \subset\{1, \ldots, t+q\}$. Тогда

$$
b * z=\sum_{J}(-1)^{\widetilde{J}}(b, z)_{J}=\sum_{n=0}^{q} \sum_{J: i_{j}=j+n}(-1)^{\widetilde{J}}(b, z)_{J} .
$$

При заданном $n$ на $(j+n)$-м месте в перетасовке $(b, z)_{J}$ стоит $b_{j}$. Поэтому для таких $J$

$$
(b, z)_{J}=\left(L_{j-1}(b), L_{n}(z)\right)_{J_{1}} \circ\left(b_{j}\right) \circ\left(R_{j}(b), R_{n}(z)\right)_{J_{2}},
$$


где

$$
\begin{aligned}
& J_{1}=\left\{i_{1}, \ldots, i_{j-1}\right\} \subset\{1, \ldots, j+n-1\}, \\
& J_{2}=\left\{i_{j+1}, \ldots, i_{t}\right\} \subset\{j+n+1, \ldots, t+q\} .
\end{aligned}
$$

При этом можно проверить, что

$$
\widetilde{J}=n(t-j+1)+\widetilde{J}_{1}+\widetilde{J}_{2}
$$

Отсюда

$$
\begin{aligned}
b * z & =\sum_{n=0}^{q} \sum_{J_{1}, J_{2}}(-1)^{n(t-j+1)+\widetilde{J}_{1}+\widetilde{J}_{2}}\left(L_{j-1}(b), L_{n}(z)\right)_{J_{1}} \circ\left(b_{j}\right) \circ\left(R_{j}(b), R_{n}(z)\right)_{J_{2}} \\
& =\sum_{n=0}^{q}(-1)^{n(t-j+1)}\left(L_{j-1}(b) * L_{n}(z)\right) \circ\left(b_{j}\right) \circ\left(R_{j}(b) * R_{n}(z)\right) .
\end{aligned}
$$

Лемма доказана.

Леммы 2.4 и 2.5 будут нами использованы для доказательства следующего предложения.

ПрЕДЛОЖЕНИЕ 2.1. Пусть $a=\left(a_{1}, \ldots, a_{t}\right) \in C_{t}(G), x=\left(x_{1}, \ldots, x_{k}\right) \in C_{k}(G)$, $y=\left(y_{1}, \ldots, y_{m}\right) \in C_{m}(G)$. Тогда

$$
[a, x * y]=[a, x] * y+(-1)^{\widetilde{x} \widetilde{y}}[a, y] * x .
$$

ДокАЗАТЕЛЬСтво. Пусть $N=\{1, \ldots, k+m\}, J=\left\{i_{1}, \ldots, i_{k}\right\} \subset N, N \backslash J=$ $\left\{j_{1}, \ldots, j_{m}\right\}$. Тогда в силу леммы 2.1

$$
[a, x * y]=\sum_{J}(-1)^{\widetilde{J}}\left[a,(x, y)_{J}\right]=\sum_{J}(-1)^{\widetilde{J}}\left(S_{a}(x, J)+S_{a}(y, J)\right),
$$

где

$$
\begin{aligned}
& S_{a}(x, J)=\sum_{l=1}^{t} \sum_{s=1}^{k}(-1)^{l+i_{s}(t-l)}\left(L_{l-1}(a) * L_{i_{s}-1}\left((x, y)_{J}\right)\right) \circ\left(\left(a_{l} x_{s}\right)-\left(x_{s} a_{l}\right)\right) \\
& \circ\left(R_{l}(a) * R_{i_{s}}\left((x, y)_{J}\right)\right), \\
& S_{a}(y, J)=\sum_{l=1}^{t} \sum_{s=1}^{m}(-1)^{l+j_{s}(t-l)}\left(L_{l-1}(a) * L_{j_{s}-1}\left((x, y)_{J}\right)\right) \circ\left(\left(a_{l} y_{s}\right)-\left(y_{s} a_{l}\right)\right) \\
& \circ\left(R_{l}(a) * R_{j_{s}}\left((x, y)_{J}\right)\right) .
\end{aligned}
$$

Покажем, что

$$
\sum_{J}(-1)^{\widetilde{J}} S_{a}(x, J)=[a, x] * y .
$$


С одной стороны, по лемме 2.4

$$
\begin{aligned}
\sum_{J}(-1)^{\widetilde{J}} S_{a}(x, J)= & \sum_{n=0}^{m} \sum_{J: i_{s}=s+n}(-1)^{\widetilde{J}} S_{a}(x, J) \\
= & \sum_{l=1}^{t} \sum_{s=1}^{k} \sum_{n=0}^{m}(-1)^{e}\left(L_{l-1}(a) * L_{s-1}(x) * L_{n}(y)\right) \\
& \circ\left(\left(a_{l} x_{s}\right)-\left(x_{s} a_{l}\right)\right) \circ\left(R_{l}(a) * R_{s}(x) * R_{n}(y)\right),
\end{aligned}
$$

где $e=l+s(t-l)+n(t-l+k-s+1)$. С другой стороны,

$[a, x]=\sum_{l=1}^{t} \sum_{s=1}^{k}(-1)^{l+s(t-l)}\left(L_{l-1}(a) * L_{s-1}(x)\right) \circ\left(\left(a_{l} x_{s}\right)-\left(x_{s} a_{l}\right)\right) \circ\left(R_{l}(a) * R_{s}(x)\right)$.

Из леммы 2.5 следует, что

$$
\begin{aligned}
{[a, x] * y=\sum_{l=1}^{t} \sum_{s=1}^{k} \sum_{n=0}^{m}(-1)^{f}\left(L_{l-1}(a) * L_{s-1}(x) * L_{n}(y)\right) } & \\
\circ\left(\left(a_{l} x_{s}\right)-\left(x_{s} a_{l}\right)\right) & \circ\left(R_{l}(a) * R_{s}(x) * R_{n}(y)\right),
\end{aligned}
$$

где $f=l+s(t-l)+n(k+t-(l+s-2)+1) \equiv e(\bmod 2)$, т.е. обе тройные суммы равны. Аналогично можно показать, что

$$
\sum_{J}(-1)^{\widetilde{J}} S_{a}(y, J)=(-1)^{\widetilde{x} \widetilde{y}}[a, y] * x .
$$

Предложение доказано.

СлеДСТвИЕ 2.3. Пусть $z \in C_{t}(G)$. Рассмотрим отображение $\operatorname{ad}(z): x \rightarrow$ $[z, x]$ степени $\widetilde{z}-1$. Тогда ад(z) является дифференцированием алгебры $C(G)$.

ДокАЗАТЕЛЬСТво. По линейности из предложения 2.1 получаем, что

$$
[z, x * y]=[z, x] * y+(-1)^{\widetilde{x} \widetilde{y}}[z, y] * x
$$

для любых однородных элементов $x, y, z \in C(G)$. Кроме того,

$$
[z, y] * x=(-1)^{(\widetilde{z}+\widetilde{y}-1) \widetilde{x}} x *[z, y] .
$$

Отсюда получаем, что $\operatorname{ad}(z)(x * y)=(\operatorname{ad}(z) x) * y+(-1)^{(\widetilde{z}-1) \widetilde{x}} x *(\operatorname{ad}(z) y)$, т.е. $a d(z)$ является дифференцированием степени $\widetilde{z}-1$.

СлЕДСТвиЕ 2.4. В $C(G)$ выполнень следующие коммутаторные тождества, в которых $x, y, z$ предполагаются однородными:

1) $[x, y]=(-1)^{\widetilde{x}} \tilde{y}[y, x]$;

2) $d[x, y]=-[d x, y]-(-1)^{\widetilde{x}}[x, d y]$;

3) $(-1)^{\widetilde{x}} \tilde{z}[[x, y], z]+(-1)^{\widetilde{y} \widetilde{x}}[[y, z], x]+(-1)^{\tilde{z} \widetilde{y}}[[z, x], y]=0$. 
ДокАЗАТЕЛЬСтво. Поскольку $[x, y]=d(x * y)-d x * y-(-1)^{\widetilde{x}} x * d y$, то первые два тождества вытекают из коммутативности перетасовочного умножения в $C(G)$ и равенства $d^{2}=0$. Третье тождество получается в результате непосредственного дифференцирования равенства

$$
[z, x * y]=[z, x] * y+(-1)^{\widetilde{x}} \widetilde{y}[z, y] * x
$$

с использованием тождеств 1) и 2).

СлЕДСТВИЕ 2.5. Для однородных $z_{1}, \ldots, z_{n} \in C(G)$ верно равенство

$$
\left[a, z_{1} * \cdots * z_{n}\right]=\sum_{p=1}^{n}(-1)^{\sigma_{p} \widetilde{z}_{p}}\left[a, z_{p}\right] * z_{1} * \cdots \widehat{z_{p}} \cdots * z_{n}
$$

əде $\sigma_{p}=\widetilde{z}_{1}+\cdots+\widetilde{z}_{p-1}$.

ДоКАЗАТЕЛЬСтво проведем индукцией по $n$. При $n=2$ формула верна в силу предложения 2.1. Пусть эта формула справедлива при некотором $n \geqslant 2$. Тогда из предложения 2.1 следует равенство

$$
\left[a, z_{1} * \cdots * z_{n+1}\right]=\left[a, z_{1} * \cdots * z_{n}\right] * z_{n+1}+(-1)^{\sigma_{n} \widetilde{z}_{n+1}}\left[a, z_{n+1}\right] * z_{1} * \cdots * z_{n},
$$

что доказывает справедливость формулы для $(n+1)$ сомножителей.

Докажем теперь основное утверждение этого параграфа.

ПрЕДЛОЖЕНИЕ 2.2. В алгебре $C(G)$ произвольной группь $G$ имеет место следующая формула для дифференцирования перетасовочного произведения $h$ однородных сомножителей:

$$
\begin{aligned}
d\left(z_{1} * \cdots * z_{h}\right)= & \sum_{1 \leqslant p \leqslant h}(-1)^{\sigma} z_{1} * \cdots d z_{p} \cdots * z_{h} \\
& +\sum_{1 \leqslant t<q \leqslant h}(-1)^{\varepsilon_{t q}}\left[z_{t}, z_{q}\right] * z_{1} * \cdots \widehat{z_{t}} \cdots \widehat{z}_{q} \cdots * z_{h}
\end{aligned}
$$

əде $\sigma_{p}=\widetilde{z}_{1}+\cdots+\widetilde{z}_{p-1}, \varepsilon_{t q}=\sigma_{t} \widetilde{z}_{t}+\sigma_{q} \widetilde{z}_{q}+\widetilde{z}_{t} \widetilde{z}_{q}$.

ДокАЗАТЕЛЬСТво. При $h=2$ формула (2.2) дает

$$
d\left(z_{1} * z_{2}\right)=d z_{1} * z_{2}+(-1)^{\tilde{z}_{1}} z_{1} * d z_{2}+\left[z_{1}, z_{2}\right]
$$

Рассмотрим $d\left(z_{1} * \cdots * z_{h+1}\right)$, предполагая, что формула верна при некотором $h \geqslant 2$. Тогда

$$
\begin{aligned}
& d\left(z_{1} * \cdots * z_{h+1}\right)=d\left(z_{1} * \cdots * z_{h}\right) * z_{h+1} \\
& \quad+(-1)^{\sigma_{h+1}} z_{1} * \cdots * z_{h} * d z_{h+1}+\left[z_{1} * \cdots * z_{h}, z_{h+1}\right] .
\end{aligned}
$$

По следствиям 2.4 и 2.5

$$
\begin{aligned}
& {\left[z_{1} * \cdots * z_{h}, z_{h+1}\right]=(-1)^{\sigma_{h+1} \widetilde{z}_{h+1}}\left[z_{h+1}, z_{1} * \cdots * z_{h}\right]} \\
& =\sum_{1 \leqslant p \leqslant h}(-1)^{\varepsilon_{p, h+1}}\left[z_{p}, z_{h+1}\right] * z_{1} * \cdots \widehat{z}_{p} \cdots * z_{h} .
\end{aligned}
$$

Таким образом, по индукции формула доказана для произвольного целого $h \geqslant 2$. 
ЗАмечаниЕ. Рассмотрим градуированную ассоциативную коммутативную алгебру $A=\bigoplus_{n \geqslant 0} A_{n}$ над некоторым ассоциативным коммутативным кольцом $K$ c 1 . Если задан дифференциал $d: A \rightarrow A$ степени (-1), то для однородных $x, y \in A$ определим коммутатор $[x, y]$ равенством $[x, y]=d(x y)-(d x) y-(-1)^{\widetilde{x}} x(d y)$.

Пусть в алгебре $A$ с дифференциалом $d$ для всех однородных $x, y, z \in A$ выполнено равенство

$$
[x, y z]=[x, y] z+(-1)^{\widetilde{y} \widetilde{z}}[x, z] y .
$$

Можно показать, что для такой пары $(A, d)$ справедливы формула дифференцирования произведения из предложения 2.2 и тождества из следствия 2.4. Примером алгебры со свойством (2.3) является, очевидно, алгебра $(C(G), d)$ для произвольной группы $G$. Другой известный пример - это внешняя алгебра $\Lambda(\mathscr{L})$ произвольной алгебры Ли $\mathscr{L}$ над кольцом $K$, дифференциал в которой задается на разложимых элементах формулой

$$
\partial\left(z_{1} \wedge \cdots \wedge z_{n}\right)=\sum_{1 \leqslant s<t \leqslant n}(-1)^{s+t-1}\left[z_{s}, z_{t}\right] \wedge z_{1} \wedge \cdots \widehat{z_{s}} \cdots \widehat{z_{t}} \cdots \wedge z_{n}
$$

где $z_{i} \in \mathscr{L}$, а $\left[z_{s}, z_{t}\right]$ - лиевский коммутатор в $\mathscr{L}$ (путаницы между двумя коммутаторами от элементов $x, y \in \Lambda^{1}(\mathscr{L})=\mathscr{L}$ не возникает, поскольку значения обоих совпадают).

Доказательство можно провести по схеме этого параграфа, но нам этот факт не понадобится.

\section{§3. Комплекс $K(G)$}

3.1. Пусть $r \geqslant 2$ - натуральное число. Рассмотрим свободную нильпотентную группу $G=G(r)$ класса 2 с $r$ свободньми порождающими $x_{1}, \ldots, x_{r}$. Мы будем пользоваться копредставлением этой группы, определенным в п. 1.1, а также теми понятиями, которые были введены в первом параграфе (геометрической интерпретацией образующих, четностью дерева и т. д.). Кроме того, будем использовать следуюшие обозначения: если $v=v_{k}$ - вершина симплекса $\Delta$, а $e=e_{i j}$ - его ребро, то $x(v)=x_{k}, c(e)=c_{i j}$.

3.2. Элементы $c(V)$ и $w(V)$. Рассмотрим максимальное дерево $T$ в $\Delta^{1}$. Зафиксируем в определенном порядке векторы $e_{1}(T), \ldots, e_{r-1}(T)$, соответствуюшие ориентированным ребрам $T$, а также произвольный вектор $a=\left(a_{1}, \ldots, a_{r}\right) \in \mathbb{R}^{r}$. Обозначим через $\left(a, e_{1}(T), \ldots, e_{r-1}(T)\right)(r \times r)$-матрицу, строки которой составлены из координат соответствующих векторов.

Лемма 3.1. Справедлива формула

$$
\operatorname{det}\left(a, e_{1}(T), \ldots, e_{r-1}(T)\right)= \pm\left(a_{1}+\cdots+a_{r}\right) .
$$

ДоКАЗАТЕЛЬСТво. Если $a_{1}+\cdots+a_{r}=0$, то $a$ линейно выражается через $e_{1}(T), \ldots, e_{r-1}(T)$, и определитель равен 0 . В противном случае все $r$ векторов образуют базис $\mathbb{R}^{r}$. При необходимости меняя местами $e_{k}(T)$ и $e_{j}(T)$, мы можем считать, что порядок $e_{1}(T), \ldots, e_{r-1}(T)$ совпадает с порядком соответствуюших им коммутаторов. В частности, $e_{r-1}(T)=e_{i r}=(0, \ldots,-1,0, \ldots, 1)-$ старшее 
ребро в дереве $T$. Прибавим к $i$-му столбцу определителя $r$-й и разложим по последней строке. Получим равенство

$$
\operatorname{det}\left(a, e_{1}(T), \ldots, e_{r-1}(T)\right)=\operatorname{det}\left(a^{\prime}, e_{1}\left(T^{\prime}\right), \ldots, e_{r-2}\left(T^{\prime}\right)\right),
$$

где $a^{\prime}=\left(a_{1}, \ldots, a_{i}+a_{r}, \ldots, a_{r-1}\right) \in \mathbb{R}^{r-1}$, а $T^{\prime}$ - некоторое максимальное дерево с $r-1$ вершиной в $\Delta^{1}(r-1)$. Поскольку утверждение леммы при $r=2$ очевидно, то ее можно считать доказанной по индукции.

Для подмножества $V \subseteq \Delta^{0}$ определим элементы $c(V) \in C_{k-1}(G)$ следуюшим образом. Пусть $\max (V)$ - множество всех максимальных деревьев $T$ в $\Gamma^{1}(V)$. Тогда

$$
c(V)=\sum_{T \in \max (V)}(-1)^{\varepsilon(T)}\left(c_{1}(T)\right) * \cdots *\left(c_{k-1}(T)\right)=\sum_{T \in \max (V)}(-1)^{\varepsilon(T)} C(T),
$$

где $c_{i}(T) \in G$ - коммутатор, соответствуюший ребру $e_{i}(T)$ дерева $T$ (напомним, что ребра в дереве $T$ берутся в порядке возрастания). Но четность дерева можно подсчитать и при любом другом порядке ребер, поменяв местами соответствующие строки в определителе, подсчитывающем эту четность. Это никак не повлияет на $c(V)$, ведь при перестановкеместами двух строк определителя и перестановке соответствуюших этим ребрам коммутаторов в перетасовочном произведении знаки меняются одновременно. Поэтому элемент

$$
(-1)^{\varepsilon(T)} C(T)=(-1)^{\varepsilon(T)}\left(c_{1}(T)\right) * \cdots *\left(c_{k-1}(T)\right) \in C_{k-1}(G)
$$

зависит только от дерева $T$.

ПримеР. Если $|V| \leqslant 1$, то $c(V)=0$, а для $V=\left\{v_{i}, v_{j}\right\}-c(V)=\left(c_{i j}\right)$. В случае, когда $V=\left\{v_{i}, v_{j}, v_{k}\right\}, c(V)=\left(c_{i j}\right) *\left(c_{i k}\right)+\left(c_{i j}\right) *\left(c_{j k}\right)+\left(c_{i k}\right) *\left(c_{j k}\right)$. Вообше, если $|V|=k \geqslant 1$, то число максимальных деревьев в множестве $\max (V)$ равно $k^{k-2}$ (формула Кәли).

Перейдем теперь к построению элементов $w(V) \in C_{k}(G)$, из которых при помощи перетасовочного произведения будет сконструирован конечньй базис (над $\mathbb{Z}$ ) некоторого подкомплекса $K(G) \subset C(G)$. По определению считаем $w(\varnothing)=($ ) $\in$ $C_{0}(G)$. При $|V| \geqslant 1$ положим

$$
w(V)=\sum_{T \in \max (V)}(-1)^{\varepsilon(T)}\left(x(V) u(T) ; c_{1}(T) * \cdots * c_{k-1}(T)\right),
$$

где $x(V)=x_{s_{k}} \cdots x_{s_{1}}$ - инверсное произведение образующих, соответствующих упорядоченному подмножеству $V=\left\{v_{s_{1}}, \ldots, v_{s_{k}}\right\}$, а $u(T)$ - коммутаторная метка дерева $T$, определенная в п. 1.4. Например,

$$
\begin{gathered}
w\left(v_{i}\right)=\left(x_{i}\right), \quad w\left(\left\{v_{i}, v_{j}\right\}\right)=\left(x_{j} x_{i}, c_{i j}\right), \\
w\left(\left\{v_{i}, v_{j}, v_{k}\right\}\right)=\left(x_{k} x_{j} x_{i} ; c_{i j} * c_{j k}\right)+\left(x_{k} x_{j} x_{i} c_{j k} ; c_{i j} * c_{i k}\right)+\left(x_{k} x_{j} x_{i} c_{i j} ; c_{i k} * c_{j k}\right) .
\end{gathered}
$$

Основньм свойством элементов $w(V)$ является то, что они удовлетворяют следующему “дифференциальному” уравнению:

$$
d w(V)=c(V)+\sum_{V=V_{1} \sqcup V_{2}}(-1)^{\varepsilon\left(V_{1}, V_{2}\right)}\left[w\left(V_{1}\right), w\left(V_{2}\right)\right]
$$

где сумма берется по всем разбиениям $V=V_{1} \sqcup V_{2}$ на два непустых подмножества, a $[\cdot, \cdot]$ - коммутатор, определенньй в п. 2.2. Доказательство этого свойства мы отложим до п. 3.4. 
3.3. Аддитивный базис комплекса $K(G)$. Для некоторого множества $V \subseteq\left\{v_{1}, \ldots, v_{r}\right\}$ рассмотрим элемент

$$
X(V)=(-1)^{|V|} \sum_{V=V_{1} \sqcup \cdots \sqcup V_{h}}(-1)^{h+\varepsilon\left(V_{1}, \ldots, V_{h}\right)} w\left(V_{1}\right) * \cdots * w\left(V_{h}\right),
$$

где сумма берется по всем разбиениям множества $V$ на непересекающиеся подмножества $V_{1}, \ldots, V_{h}$. Порядок $V_{1}, \ldots, V_{h}$ в разбиении может быть произвольным, но перетасовочное произведение должно быть согласовано с этим порядком. При перестановке местами $V_{i}$ и $V_{j}$ четность разбиения меняется на $\left|V_{i}\right|\left|V_{j}\right|(\bmod 2)$, перестановка множителей $w\left(V_{i}\right)$ и $w\left(V_{j}\right)$ меняет четность на ту же величину, так что само слагаемое оказывается не зависящим от порядка подмножеств в разбиении. Отметим, что если $|V|=k$, то число слагаемых в сумме равно числу Белла $B(k)$. В частности, $B(1)=1, B(2)=2, B(3)=5, \ldots$.

ЛЕмма 3.2. Для әлементов $X(V) \in C_{|V|}(G)$ выполнено следующее равенство:

$$
d X(V)=\sum_{P \subseteq V}(-1)^{\varepsilon(P, V \backslash P)+|P|-1} c(P) * X(V \backslash P) .
$$

ДокаЗАТЕЛЬСтво. Для дифференцирования $X(V)$ используем формулу (2.2) и равенство (3.1). Получим, что $d X(V)=S_{1}+S_{2}+S_{3}$, где первое слагаемое равно

$$
\begin{aligned}
& \sum_{V=V_{1} \sqcup \cdots \sqcup V_{h}}(-1)^{|V|+h+\varepsilon\left(V_{1}, \ldots, V_{h}\right)} \sum_{p=1}^{h}(-1)^{\sigma_{p}} w\left(V_{1}\right) * \cdots * c\left(V_{p}\right) * \cdots * w\left(V_{h}\right), \\
& \sigma_{p}=\left|V_{1}\right|+\cdots+\left|V_{p-1}\right|, \text { второе - } \\
& \quad \sum_{V=V_{1} \sqcup \cdots \sqcup V_{h}} \sum_{p=1}^{h} \sum_{V_{p}=V_{p}^{\prime} \sqcup V_{p}^{\prime \prime}}(-1)^{f} w\left(V_{1}\right) * \cdots *\left[w\left(V_{p}^{\prime}\right), w\left(V_{p}^{\prime \prime}\right)\right] * \cdots * w\left(V_{h}\right), \\
& f=|V|+h+\varepsilon\left(V_{1}, \ldots, V_{h}\right)+\sigma_{p}+\varepsilon\left(V_{p}^{\prime}, V_{p}^{\prime \prime}\right), \text { и наконец, третье - } \\
& \quad \sum_{V=V_{1} \sqcup \cdots \sqcup V_{h}} \sum_{1 \leqslant t<q \leqslant h}(-1)^{m}\left[w\left(V_{t}\right), w\left(V_{q}\right)\right] * w\left(V_{1}\right) \cdots \widehat{w\left(V_{t}\right)} \cdots \widehat{w\left(V_{q}\right)} \cdots w\left(V_{h}\right), \\
& m=|V|+h+\varepsilon\left(V_{1} \ldots, V_{h}\right)+\sigma_{t}\left|V_{t}\right|+\sigma_{q}\left|V_{q}\right|+\left|V_{t}\right|\left|V_{q}\right| .
\end{aligned}
$$

Покажем сначала, что $S_{2}+S_{3}=0$ (фактически уравнение (3.1) как раз и обеспечивает это равенство). Для этого во второй сумме рассмотрим слагаемое

$$
(-1)^{f} w\left(V_{1}\right) * \cdots\left[w\left(V_{p}^{\prime}\right), w\left(V_{p}^{\prime \prime}\right)\right] \cdots * w\left(V_{h}\right)
$$

В сумме $S_{3}$ ему соответствует слагаемое

$$
(-1)^{m}\left[w\left(V_{p}^{\prime}\right), w\left(V_{p}^{\prime \prime}\right)\right] * w\left(V_{1}\right) \cdots \widehat{w\left(V_{p}^{\prime}\right)} \cdots \widehat{w\left(V_{p}^{\prime \prime}\right)} \cdots w\left(V_{h}\right)
$$


из разбиения $\bar{V}=V_{1} \sqcup \cdots \sqcup V_{p}^{\prime} \sqcup V_{p}^{\prime \prime} \sqcup \cdots \sqcup V_{h}$. При этом

$$
\begin{aligned}
m & =|V|+h+1+\varepsilon\left(V_{1}, \ldots, V_{p}^{\prime}, V_{p}^{\prime \prime}, \ldots, V_{h}\right)+\left|V_{p}^{\prime}\right| \sigma_{p}+\left|V_{p}^{\prime \prime}\right|\left(\sigma_{p}+\left|V_{p}^{\prime}\right|\right)+\left|V_{p}^{\prime}\right|\left|V_{p}^{\prime \prime}\right| \\
& \equiv|V|+h+1+\varepsilon\left(V_{1}, \ldots, V_{p}, \ldots, V_{h}\right)+\varepsilon\left(V_{p}^{\prime}, V_{p}^{\prime \prime}\right)+\left(\left|V_{p}^{\prime}\right|+\left|V_{p}^{\prime \prime}\right|\right) \sigma_{p} \\
& \equiv 1+f+\sigma_{p}\left(\left|V_{p}\right|-1\right)(\bmod 2) .
\end{aligned}
$$

Таким образом, все слагаемые в $S_{2}$ и $S_{3}$ попарно сокрашаются, т.е. $d X(V)=S_{1}$. Для получения окончательной формулы рассмотрим все разбиения, которые содержат некоторое непустое фиксированное подмножество $P \subseteq V$. Можно считать, что $P=V_{1}$. Тогда

$$
S_{1}=\sum_{P \subseteq V} \sum_{V \backslash P=V_{2} \sqcup \cdots \sqcup V_{h}}(-1)^{l} c(P) * w\left(V_{2}\right) * \cdots * w\left(V_{h}\right),
$$

где $l=|V|+h+1+\varepsilon\left(P, V_{2}, \ldots, V_{h}\right)$. Внутренняя сумма дает

$$
(-1)^{|P|-1+\varepsilon(P, V \backslash P)} c(P) * X(V \backslash P),
$$

поскольку

$$
\begin{aligned}
|V|+h & +1+\varepsilon\left(P, V_{2}, \ldots, V_{h}\right) \\
& \equiv|P|-1+\varepsilon(P, V \backslash P)+\varepsilon\left(V_{2}, \ldots, V_{h}\right)+h-1+|V|-|P|(\bmod 2) .
\end{aligned}
$$

Тем самым лемма доказана.

Пусть $\Delta^{1}=\Delta^{1}(r)$ - упорядоченное множество ориентированњшх ребер симплекса $\Delta$, т.е. $\Delta^{1}=\left\{e_{i j}, 1 \leqslant i<j \leqslant r\right\}$. Если $E=\left\{e_{i_{1} j_{1}}, \ldots, e_{i_{q} j_{q}}\right\}$, то положим

$$
C(E)=\left(c_{i_{1} j_{1}}\right) * \cdots *\left(c_{i_{q} i_{q}}\right) .
$$

В частности, $C(\varnothing)=\left(\right.$ ). Для всевозможных $E \subseteq \Delta^{1}, V \subseteq \Delta^{0}$ рассмотрим элементы $C(E) * X(V)$. По следствию 2.2 и лемме 3.2

$$
d(C(E) * X(V))=(-1)^{|E|} C(E) * d X(V)
$$

можно представить в виде линейной комбинации определенных элементов $C\left(E^{\prime}\right) * X\left(V^{\prime}\right)$, т.е. линейные комбинации элементов вида $C(E) * X(V)$ составляют некоторьй подкомплекс $K(G)$ в групповом комплексе $C(G)$.

Покажем, что элементы $C(E) * X(V)$ образуют базис в $K(G)$. Запишем $C(E) * X(V)$ в виде

$$
\left(c_{i_{1} j_{1}}\right) * \cdots *\left(c_{i_{q} j_{q}}\right) *\left(x_{s_{1}}\right) * \cdots *\left(x_{s_{k}}\right)+\cdots,
$$

где многоточие означает слагаемые, отвечающие разбиениям $V$ на подмножества, среди которых хотя бы одно имеет не менее двух элементов. Очевидно, что первые слагаемые в сумме (3.3) линейно независимы для разных пар $(E, V)$, и следовательно, $C(E) * X(V)$ тоже линейно независимы.

Пусть $\mathscr{G}=G(r)$ - свободное нильпотентное кольцо Ли класса $2 \mathrm{c} r$ свободными порождающими:

$$
\mathscr{G}=\left\langle\bar{x}_{i}, \bar{c}_{i j}, 1 \leqslant i<j \leqslant r \mid \bar{c}_{i j}=\left[\bar{x}_{i}, \bar{x}_{j}\right],\left[\bar{c}_{i j}, \bar{x}_{k}\right]=\left[\bar{c}_{i j}, \bar{c}_{k l}\right]=0\right\rangle .
$$

Для $\bar{x}_{i}$ и $\bar{c}_{i j}$ можно использовать ту же самую геометрическую интерпретацию, что и в групповом случае. Рассмотрим внешнюю алгебру $\Lambda(\mathscr{G})$. Пусть $E=$ $\left\{e_{i_{1} j_{1}}, \ldots, e_{i_{q} j_{q}}\right\} \subseteq \Delta^{1}, V=\left\{v_{s_{1}}, \ldots, v_{s_{k}}\right\} \subseteq \Delta^{0}-$ те же подмножества, что и вьше. Обозначим

$$
\begin{gathered}
\bar{X}(V)=\bar{x}_{s_{1}} \wedge \cdots \wedge \bar{x}_{s_{k}} \in \Lambda^{k}(G), \quad \bar{C}(E)=\bar{c}_{i_{1} j_{1}} \wedge \cdots \wedge \bar{c}_{i_{q} j_{q}} \in \Lambda^{q}(G), \\
\bar{X}(\varnothing)=\bar{C}(\varnothing)=1 \in \Lambda^{0}(G)=\mathbb{Z} .
\end{gathered}
$$




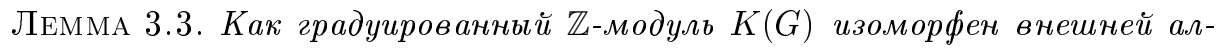
гебре $\Lambda(\mathscr{G})$.

ДокАЗАТЕЛЬСтво. Базис алгебры $\Lambda(\mathscr{G})$ образуют элементы вида $\bar{C}(E) \wedge \bar{X}(V)$. Поэтому изоморфизм $f: K(G) \stackrel{\sim}{\rightarrow} \Lambda(\mathscr{G})$ можно задать соответствием

$$
C(E) * X(V) \rightarrow(-1)^{|E|} \bar{C}(E) \wedge \bar{X}(V) .
$$

Отождествив при помоши изоморфизма $f$ градуированные $\mathbb{Z}$-модули $K(G)$ и $\Lambda(\mathscr{G})$, мы получим комплекс $(\Lambda(\mathscr{G}), d)$ (дифференциал, по-прежнему, обозначается той же буквой). Из равенства

$$
d(C(E)) * X(V)=(-1)^{|E|} C(E) * d X(V)
$$

и леммы 3.2. можно заключить, что дифференциал $d$ определяется следующими свойствами:

1) $d \bar{C}(E) \wedge \bar{X}(V)=(-1)^{|E|} \bar{C}(E) \wedge d \bar{X}(V)$

2) $d \bar{X}(V)=\sum_{P \subseteq V}(-1)^{\varepsilon(P, V \backslash P)} \bar{c}(P) \wedge \bar{X}(V \backslash P)$, где

$$
\bar{c}(P)=\sum_{T \in \max (P)}(-1)^{\varepsilon(T)} \bar{c}_{1}(T) \wedge \cdots \wedge \bar{c}_{k-1}(T), \quad k=|P| .
$$

В частности, дифференциал $d$ можно представить в виде суммы $d_{2}+d_{3}+\cdots+d_{r}$, в которой

$$
d_{k} \bar{X}(V)=\sum_{P \subseteq V,|P|=k}(-1)^{\varepsilon(P, V \backslash P)} \bar{c}(P) \wedge \bar{X}(V \backslash P)
$$

$\left(d_{k}\right.$ удовлетворяет и первому свойству дифференциала $d$.)

ЗАмечАниЕ. Можно показать, что выполнены следуюшие соотношения между $d_{k}: d_{k}^{2}=0$ и $d_{i} d_{j}+d_{j} d_{i}=0$ для всех $i, j, k$ от 2 до $r$.

\section{4. Коммутаторные метки деревьев.}

3.4.1. Покажем, что элементы $w(V)$ удовлетворяют уравнению (3.1). Подставим выражения для $w(V), w\left(V_{1}\right)$ и $w\left(V_{2}\right)$ в $(3.1)$ и затем применим леммы $2.2,2.3$. Получим уравнение

$$
\begin{aligned}
\sum_{T} & \sum_{p=1}^{k-1}(-1)^{\varepsilon(T)+p-1}\left(x(V) u(T) ; c_{1}(T) * \cdots \widehat{c_{p}(T)} \cdots * c_{k-1}(T)\right) \\
& -\sum_{T} \sum_{p=1}^{k-1}(-1)^{\varepsilon(T)+p-1}\left(x(V) u(T) c_{p}(T) ; c_{1}(T) * \cdots \widehat{c_{p}(T)} \cdots * c_{k-1}(T)\right) \\
= & \sum_{V=V_{1} \sqcup V_{2}} \sum_{T_{1}, T_{2}}(-1)^{\mu}\left(x\left(V_{1}\right) x\left(V_{2}\right) u\left(T_{1}\right) u\left(T_{2}\right) ; c_{1}\left(T_{1}\right) * \cdots * c_{1}\left(T_{2}\right) * \cdots\right) \\
& -\sum_{V=V_{1} \sqcup V_{2}} \sum_{T_{1}, T_{2}}(-1)^{\mu}\left(x\left(V_{2}\right) x\left(V_{1}\right) u\left(T_{1}\right) u\left(T_{2}\right) ; c_{1}\left(T_{1}\right) * \cdots * c_{1}\left(T_{2}\right) * \cdots\right) .
\end{aligned}
$$


В этом равенстве $\mu=\varepsilon\left(T_{1}\right)+\varepsilon\left(T_{2}\right)+\varepsilon\left(V_{1}, V_{2}\right)+\left|V_{1}\right|, T$ пробегает множество $\max (V), T_{1}$ и $T_{2}-\max \left(V_{1}\right)$ и $\max \left(V_{2}\right)$, соответственно.

Чтобы упростить равенство (3.4), рассмотрим более подробно левую и правую части. Двойную сумму слева можно интерпретировать следующим образом: мы берем дерево $T$ и последовательно удаляем из него по одному ребру. При удалении одного ребра дерево $T$ распадается на два поддерева $T_{1}$ и $T_{2}$, вершины которых образуют разбиение $V=V_{1} \sqcup V_{2}$. Именно эти два поддерева и фигурируют в правой части. Зафиксируем разбиение $V=V_{1} \sqcup V_{2}$ (для определенности будем считать, что наибольший элемент из $V$ лежит в $\left.V_{2}\right)$, и пусть $T_{1} \in \max \left(V_{1}\right), T_{2} \in \max \left(V_{2}\right)$. Другими словами, фиксирован элемент суммы в правой части (3.4). Какие деревья $T$ из левой части дают при удалении ребра деревья $T_{1}$ и $T_{2}$ ? Очевидно те, которые получаются из $T_{1}$ и $T_{2}$ добавлением ребра, одна вершина которого лежит в $T_{1}$, другая - в $T_{2}$. Обозначим множество таких ребер $\mathscr{E}\left(T_{1}, T_{2}\right)$. При этом $\left|\mathscr{E}\left(T_{1}, T_{2}\right)\right|=\left|V_{1}\right|\left|V_{2}\right|=t$. Для нас будет важно, что $\mathscr{E}\left(T_{1}, T_{2}\right)$ состоит из ребер двух типов: выходящих из $T_{1}$ (их множество обозначим $\mathscr{E}_{+}\left(T_{1}, T_{2}\right)$ ) и выходящих из $T_{2}$ (соответственно, $\left.\mathscr{E}_{-}\left(T_{1}, T_{2}\right)\right)$, т.е. $\mathscr{E}\left(T_{1}, T_{2}\right)=\mathscr{E}_{+}\left(T_{1}, T_{2}\right) \sqcup \mathscr{E}_{-}\left(T_{1}, T_{2}\right)$. Обозначим $T_{1} \#{ }_{e} T_{2}$ дерево $T$, которое получается добавлением к $T_{1}, T_{2}$ ребра $e$. Предположим, что для любой пары $T_{1}, T_{2}$ выполняется равенство ( $\mu$ - то же, что и в (3.4))

$$
\begin{aligned}
& \sum_{T=} T_{1} \#_{e} T_{2} \\
& -(-1)^{\varepsilon(T)}\left(\left(x(V) u(T) c(e) ; c_{2}(T) * \cdots * c_{k-1}(T)\right)\right) \\
= & (-1)^{\mu}\left(\left(x\left(V_{2}\right) x\left(V_{1}\right) u\left(T_{1}\right) u\left(T_{2}\right) ; c_{1}\left(T_{1}\right) * \cdots * c_{1}\left(T_{2}\right) * \cdots\right)\right. \\
& \left.-\left(x\left(V_{2}\right) x\left(V_{1}\right) u\left(T_{1}\right) u\left(T_{2}\right) ; c_{1}\left(T_{1}\right) * \cdots * c_{1}\left(T_{2}\right) * \cdots\right)\right) .
\end{aligned}
$$

В левой части (3.5) суммирование производится по всем ребрам $e \in \mathscr{E}\left(T_{1}, T_{2}\right)$. При этом мы считаем, что $c_{1}(T)=c(e)$, т.е. ребро е удаляется первым. Ясно, что при суммировании равенств типа (3.5) по всем парам $T_{1}, T_{2}$ получается в точности равенство (3.4). Поэтому достаточно доказать справедливость (3.5) для произвольной пары деревьев $T_{1}, T_{2}$. Сделаем еще одно упрощение. В (3.5) все перетасовочные произведения коммутаторов одинаковы с точностью до перестановки множителей. Мы будем считать, что в каждом слагаемом из левой части порядок коммутаторов такой же, как в правой. В соответствии с этим подсчитывается и четность $\varepsilon(T)$. Кроме того, рассмотрим инверсные произведения. Пользуясь соотношениями в группе $G$, легко получить, что

$$
x\left(V_{1}\right) x\left(V_{2}\right)=x(V) \prod_{e \in \mathscr{E}_{+}\left(T_{1}, T_{2}\right)} c(e), \quad x\left(V_{2}\right) x\left(V_{1}\right)=x(V) \prod_{e \in \mathscr{E}_{-}\left(T_{1}, T_{2}\right)} c(e) .
$$

Если показать, что в групповом кольце $\mathbb{Z} G$

$$
\begin{aligned}
\sum_{T=T_{1} \#_{e} T_{2}}(-1)^{\varepsilon(T)+\varepsilon\left(T_{1}\right)+\varepsilon\left(T_{2}\right)+\varepsilon\left(V_{1}, V_{2}\right)+\left|V_{1}\right|}(u(T)-u(T) c(e)) & \\
& =u\left(T_{1}\right) u\left(T_{2}\right) \prod_{\mathscr{E}_{+}\left(T_{1}, T_{2}\right)} c(e)-u\left(T_{1}\right) u\left(T_{2}\right) \prod_{\mathscr{E}_{-}\left(T_{1}, T_{2}\right)} c(e),
\end{aligned}
$$

причем в левой части происходит сокрашение всех слагаемых, кроме двух, то равенство (3.5) будет установлено. 
ЛЕмма 3.4. В левой части (3.6)

$$
(-1)^{\varepsilon(T)+\varepsilon\left(T_{1}\right)+\varepsilon\left(T_{2}\right)+\varepsilon\left(V_{1}, V_{2}\right)+\left|V_{1}\right|}= \pm 1,
$$

причем “-1” получается тогда и только тогда, когда е $\in \mathscr{E}_{+}\left(T_{1}, T_{2}\right)$.

ДокАЗАтЕЛьство. Можно считать, что $V=\left\{v_{1}, \ldots, v_{k}\right\}$. Пусть $e=e_{i j}$, $i<j$. Если $V_{1}=\left\{v_{i_{1}}, \ldots, v_{i_{p}}\right\}, V_{2}=\left\{v_{j_{1}}, \ldots, v_{j_{q}}\right\}, v_{i} \in V_{1}, v_{j} \in V_{2}$ (т.е. ребро $e$ идет из $T_{1}$ в $\left.T_{2}\right), a_{i}=(0, \ldots, 1,0, \ldots, 0) \in \mathbb{R}^{k}$ (1 стоит на $i$-м месте), то по лемме 3.1

$$
\operatorname{det}\left(a_{i}, e_{i j}, e_{1}\left(T_{1}\right), \ldots, e_{1}\left(T_{2}\right), \ldots\right)=(-1)^{\varepsilon(T)}
$$

$\mathrm{C}$ другой стороны, $(-1)^{\varepsilon(T)}=\operatorname{det}\left(a_{i}, a_{i}+e_{i j}, e_{1}\left(T_{1}\right), \ldots, e_{1}\left(T_{2}\right), \ldots\right)$. Используя теорему Лапласа о разложении определителя по выделенным столбцам с номерами $i_{1}, \ldots, i_{p}$ (в этих столбцах имеется только один ненулевой минор) получим, что

$$
(-1)^{\varepsilon(T)}=(-1)^{\varepsilon\left(T_{1}\right)+\varepsilon\left(T_{2}\right)+\varepsilon\left(V_{1}, V_{2}\right)+p-1}, \quad \text { если } \quad v_{i} \in V_{1}, \quad v_{j} \in V_{2} .
$$

Второй случай разбирается аналогично.

Лемма 3.4 позволяет переписать уравнение (3.6) в следующем виде:

$$
\begin{aligned}
& \sum_{e \in \mathscr{E}\left(T_{1}, T_{2}\right)}(-1)^{\widetilde{e}}\left(u\left(T_{1} \# e_{e} T_{2}\right)-u\left(T_{1} \# e_{e} T_{2}\right) c(e)\right) \\
&=u\left(T_{1}\right) u\left(T_{2}\right) \prod_{e \in \mathscr{E}_{+}\left(T_{1}, T_{2}\right)} c(e)-u\left(T_{1}\right) u\left(T_{2}\right) \prod_{e \in \mathscr{E}_{-}\left(T_{1}, T_{2}\right)} c(e)
\end{aligned}
$$

где $\widetilde{e}=1$, если $e \in \mathscr{E}_{+}\left(T_{1}, T_{2}\right)$, и $\widetilde{e}=0$, если $e \in \mathscr{E}_{-}\left(T_{1}, T_{2}\right)$.

Отметим, что в силу определения $u(T)$ любую метку $u\left(T_{1} \# T_{2}\right)$ можно представить как произведение $u\left(T_{1}\right) u\left(T_{2}\right) y(e)$, где $y(e)$ состоит из произведения коммутаторов, соответствующих только ребрам из $\mathscr{E}\left(T_{1}, T_{2}\right)$, т.е. $u\left(T_{1} \# e_{2}\right)$ всегда “делится" на $u\left(T_{1}\right) u\left(T_{2}\right)$. Сократив последнее равенство на $u\left(T_{1}\right) u\left(T_{2}\right)$, получим

$$
\sum_{e \in \mathscr{E}_{\left(T_{1}, T_{2}\right)}}(-1)^{\widetilde{e}}(y(e)-y(e) c(e))=\prod_{e \in \mathscr{E}_{+}\left(T_{1}, T_{2}\right)} c(e)-\prod_{e \in \mathscr{E}_{-}\left(T_{1}, T_{2}\right)} c(e) .
$$

3.4.2. Упорядочение множества $\mathscr{E}\left(T_{1}, T_{2}\right)$. Пусть $e, f \in \mathscr{E}\left(T_{1}, T_{2}\right)$ и $Z=$ $Z(e, f)$ - цикл, который получается присоединением к $T_{1}, T_{2}$ ребер $e, f$. Скажем, что $e \prec f$, если при положительном обходе $Z$ после прохождения ребра $e$ мы оказываемся в дереве $T_{2}$. Если это не так, то будем говорить, что $e \succ f$.

Покажем, что введенное отношение действительно является отношением порядка на множестве $\mathscr{E}\left(T_{1}, T_{2}\right)$, т.е. если $e \prec f, f \prec h$, то и $e \prec h$. Предположим, что $h \prec e$. Рассмотрим объединение всех ребер из $Z(e, f), Z(f, h), Z(h, e)$. Это - некоторьй граф̆ $\Phi=\Phi(e, f, h)$, все вершины которого, кроме двух степени 3 , имеют степень 2 , причем граф $\Phi$ остается связньм при удалении произвольного ребра. Любое ребро $b$ граф̆а $\Phi$ входит ровно в два из вышеуказанных трех циклов. Если $e \prec f, f \prec h, h \prec e$, то при последовательном положительном обходе всех трех циклов ребро $b$ будет проходиться ровно два раза: один раз в соответствии с ориентацией ребра $b$, другой раз - в противоположном направлении. Пусть $b$ - старшее 
ребро в графе $\Phi$ (относительно порядка всех ребер симплекса $\Delta$ ). Очевидно, что $b$ - старшее ребро в обоих циклах, которым оно принадлежит. Поскольку положительный обход цикла всегда происходит в направлении старшего ребра цикла, то мы получаем противоречие со сделанным предположением, т.е. $e \prec h$.

Таким образом, можно упорядочить все ребра из $\mathscr{E}\left(T_{1}, T_{2}\right)$. Вьпишем их в порядке возрастания: $e_{1} \prec \cdots \prec e_{t}$. Обозначим

$$
\begin{gathered}
y_{-}(e)=\prod_{\substack{f \succ e \\
f \in \mathscr{E}_{-}\left(T_{1}, T_{2}\right)}} c(f), \\
y_{+}(e)=\prod_{\substack{f \prec e \\
f \in \mathscr{E}_{+}\left(T_{1}, T_{2}\right)}} c(f) .
\end{gathered}
$$

В левой части равенства (3.7) $y(e)=y_{-}(e) y_{+}(e)$. Действительно, если ребро $e$ фиксировано (скажем, $e \in \mathscr{E}_{+}\left(T_{1}, T_{2}\right)$ ), то $c(f)$ входит в метку $y(e)$ в двух случаях - а именно, если $f \succ e, f \in \mathscr{E}_{-}\left(T_{1}, T_{2}\right)$ или $f \prec e, f \in \mathscr{E}_{+}\left(T_{1}, T_{2}\right)$ (в этих случаях ребро $f$ положительно ориентировано относительно возникающего цикла $Z=Z(e, f))$. Полученное разложение $y(e)$ будем называть полярным.

Доказательство того факта, что метки $y(e)$ удовлетворяют (3.7), вытекает из следующей леммы.

ЛЕммА 3.5. Пусть

$$
S(p)=\sum_{i=1}^{p}(-1)^{\tilde{e}_{i}}\left(y\left(e_{i}\right)-y\left(e_{i}\right) c\left(e_{i}\right)\right), \quad p=1, \ldots, t .
$$

Тогда если $e_{p} \in \mathscr{E}_{+}\left(T_{1}, T_{2}\right)$, то

$$
S(p)=-\prod_{e \in \mathscr{E}_{-}\left(T_{1}, T_{2}\right)} c(e)+y\left(e_{p}\right) c\left(e_{p}\right),
$$

а если $e_{p} \in \mathscr{E}_{-}\left(T_{1}, T_{2}\right)$, mo

$$
S(p)=-\prod_{e \in \mathscr{E}_{-}\left(T_{1}, T_{2}\right)} c(e)+y\left(e_{p}\right)
$$

ДоКАЗАТЕЛЬСТВО проведем индукцией по $p$. При $p=1$ утверждение непосредственно следует из равенства $y\left(e_{1}\right)=y_{-}\left(e_{1}\right)$. Пусть уже доказано, что

$$
S(p)=-\prod_{e \in \mathscr{E}_{-}\left(T_{1}, T_{2}\right)} c(e)+y\left(e_{p}\right) c\left(e_{p}\right)
$$

(для определенности рассмотрим случай $e_{p} \in \mathscr{E}_{+}\left(T_{1}, T_{2}\right)$, оставшийся случай рассматривается аналогично). Если $e_{p+1} \in \mathscr{E}_{+}\left(T_{1}, T_{2}\right)$, то

$$
S(p+1)=-\prod_{e \in \mathscr{E}_{-}\left(T_{1}, T_{2}\right)} c(e)+y\left(e_{p}\right) c\left(e_{p}\right)-y\left(e_{p+1}\right)+y\left(e_{p+1}\right) c\left(e_{p+1}\right) .
$$

Из полярного разложения $y(e)$ легко вытекает, что $y\left(e_{p}\right) c\left(e_{p}\right)=y\left(e_{p+1}\right)$, т.е. шаг индукции сделан. Аналогично исследуется случай, когда $e_{p+1} \in \mathscr{E}_{-}\left(T_{1}, T_{2}\right)$. Лемма доказана.

При $p=t$ во всех случаях получается равенство (3.7), т.е. сушествование подкомплекса $K(G)$. 
ЗАмЕчАнИЕ. Метки $u(T)$ можно определить не единственным образом (например, если задать положительную ориентацию циклов в направлении младиего ребра), из чего следует сушествование нескольких подкомплексов вида $K(G)$. Однако, все они будут изоморфны.

\section{§4. Конечная резольвента}

В этом параграфе будет завершено доказательство теоремы 1. Конструкции предыдущего параграфа будут использованы нами для построения конечной резольвенты тривиального модуля $\mathbb{Z}$ над $\mathbb{Z} G$ для свободной нильпотентной группы $G$ класса 2.

4.1. Сначала рассмотрим произвольную группу $G$. Стандартная свободная резольвента тривиального модуля $\mathbb{Z}$ над $\mathbb{Z} G$ - это комплекс левых $\mathbb{Z} G$-модулей:

$$
\cdots \stackrel{d}{\longrightarrow} \mathbb{Z} G \otimes C_{n}(G) \stackrel{d}{\longrightarrow} \mathbb{Z} G \otimes C_{n-1}(G) \stackrel{d}{\longrightarrow} \cdots,
$$

где дифференциал $d$ является $G$-гомоморфизмом и действует на базисных элементах $1 \otimes\left(g_{1}, \ldots, g_{n}\right)$ следуюшим образом:

$$
\begin{aligned}
& d\left(1 \otimes\left(g_{1}, \ldots, g_{n}\right)\right)=g_{1} \otimes\left(g_{2}, \ldots, g_{n}\right) \\
& \quad+\sum_{p=1}^{n-1}(-1)^{p} 1 \otimes\left(g_{1}, \ldots, g_{p} g_{p+1}, \ldots, g_{n}\right)+(-1)^{n} 1 \otimes\left(g_{1}, \ldots, g_{n-1}\right) .
\end{aligned}
$$

Можно также записать, что

$$
d\left(1 \otimes\left(g_{1}, \ldots, g_{n}\right)\right)=\delta\left(1 \otimes\left(g_{1}, \ldots, g_{n}\right)\right)+1 \otimes d\left(g_{1}, \ldots, g_{n}\right),
$$

где $\delta\left(1 \otimes\left(g_{1}, \ldots, g_{n}\right)\right)=\left(g_{1}-1\right) \otimes\left(g_{2}, \ldots, g_{n}\right)$, а в правой части $d-$ это дифференциал в стандартном комплексе $C(G)$.

Пусть теперь $G=G(r)$ - свободная нильпотентная группа класса 2. Покажем, что градуированный $\mathbb{Z} G$-подмодуль $\mathbb{Z} G \otimes K(G) \subset \mathbb{Z} G \otimes C(G)$ является подкомnлексом относительно $d$. Предварительно докажем следующие леммы.

Лемма 4.1. Пусть $u_{p}=\left(u_{p 1}, \ldots, u_{p n_{p}}\right) \in C_{n_{p}}(G), p=1, \ldots, h$. Тогда

$$
\delta\left(1 \otimes u_{1} * \cdots * u_{h}\right)=\sum_{p=1}^{h}(-1)^{\sigma_{p}}\left(u_{p 1}-1\right) \otimes u_{1} * \cdots * R_{1}\left(u_{p}\right) * \cdots * u_{h},
$$

əде $R_{1}\left(u_{p}\right)=\left(u_{p 2}, \ldots, u_{p n_{p}}\right), \sigma_{p}=\widetilde{u}_{1}+\cdots+\widetilde{u}_{p-1}$.

ДокАЗАТЕльСТво. По определению перетасовочного произведения первыми элементами любой строки, входящей слагаемым в развернутое выражение для $u_{1} * \cdots * u_{h}$, могут быть только $u_{11}, \ldots, u_{h 1}$. Сумма всех слагаемых, у которых на первом месте стоит $u_{p 1}$, равна, как показывает несложный подсчет,

$$
U_{p}=(-1)^{\sigma_{p}}\left(u_{p 1}\right) \circ\left(u_{1} * \cdots * R_{1}\left(u_{p}\right) * \cdots * u_{h}\right) .
$$

Применяя $\delta$ к $1 \otimes U_{p}$ и затем суммируя по всем $p$, получим требуемую формулу. 
Лемма 4.2. Пусть $E=\left\{e_{1}, \ldots, e_{q}\right\} \subseteq \Delta^{1}, V \subseteq \Delta^{0}$. Тогда

$$
\begin{aligned}
\delta(1 \otimes C(E) & * X(V))=\sum_{i=1}^{q}(-1)^{i-1}\left(c\left(e_{i}\right)-1\right) \otimes C\left(E \backslash e_{i}\right) * X(V) \\
& +\sum_{P \subseteq V} \sum_{T \in \max (V)}(-1)^{\mu}(x(P) u(T)-1) \otimes C(E) * C(T) * X(V \backslash P),
\end{aligned}
$$

$\mu=|E|+\varepsilon(P, V \backslash P)+|P|-1+\varepsilon(T)$.

ДоКАЗАТЕЛЬСТво. Поскольку

$$
1 \otimes C(E) * X(V)=\sum_{V=V_{1} \sqcup \cdots \sqcup V_{h}}(-1)^{\lambda} 1 \otimes\left(c\left(e_{1}\right)\right) * \cdots * w\left(V_{1}\right) * \cdots * w\left(V_{h}\right),
$$

$\lambda=|V|+h+\varepsilon\left(V_{1}, \ldots, V_{h}\right)$, можно применить лемму 4.1. Сумма тех слагаемых из $\delta(1 \otimes C(E) * X(V))$, которая получается при действии $\delta$ на коммутаторную часть $1 \otimes C(E) * X(V)$, равна

$$
\sum_{i=1}^{q}(-1)^{i-1}\left(c\left(e_{i}\right)-1\right) \otimes C\left(E \backslash e_{i}\right) * X(V) .
$$

Чтобы подсчитать оставшуюся сумму, воспользуемся приемом, который уже применялся при доказательстве леммы 3.2. Пусть $P \subseteq V$ фиксировано. Считая, что $P=V_{1},|P|=k$, рассмотрим те слагаемые, которые получаются при применении оператора $R_{1}$ из леммы 4.1 к каждому слагаемому в

$$
\sum_{T \in \max (P)}(-1)^{\varepsilon(T)}\left(x(P) u(T) ; c_{1}(T) * \cdots * c_{k-1}(T)\right)=w(P) .
$$

В результате эта часть суммы оказьвается равной

$$
\sum_{T \in \max (P)}(-1)^{|E|+\varepsilon(P, V \backslash P)+|P|-1+\varepsilon(T)}(x(P) u(T)-1) \otimes C(E) * C(T) * X(V \backslash P) .
$$

Суммирование по всем $P \subseteq V$ дает окончательный ответ.

Из лемм 3.2 и 4.2 непосредственно вытекает

ПРЕДЛОЖЕНИЕ 4.1. Градуированный подмодуль $\mathbb{Z} G \otimes K(G)$ является подкомплексом в $\mathbb{Z} G \otimes C(G)$, причем ограничение дифференциала $d$ на $\mathbb{Z} G \otimes K(G)$ равно сумме $d_{0}+d_{1}+d_{\geqslant 2}$, где действие каждого слагаемого определяется следующим образом $\left(E=\left\{e_{1}, \ldots, e_{q}\right\}, V=\left\{v_{s_{1}}, \ldots, v_{s_{p}}\right\}\right)$ :

1) $d_{0}(1 \otimes C(E) * X(V))=\sum_{i=1}^{q}(-1)^{i-1}\left(c\left(e_{i}\right)-1\right) \otimes C\left(E \backslash e_{i}\right) * X(V)$;

2) $d_{1}(1 \otimes C(E) * X(V))=\sum_{j=1}^{p}(-1)^{|E|+j-1}\left(x\left(v_{j}\right)-1\right) \otimes C(E) * X\left(V \backslash v_{j}\right)$;

3) $d_{\geqslant 2}(1 \otimes C(E) * X(V))=\sum_{\substack{P \subseteq V,|P| \geqslant 2 \\ T \in \max (P)}}(-1)^{\mu} x(P) u(T) \otimes C(E) * C(T) * X(V \backslash P)$,

$$
\mu=|E|+\varepsilon(P, V \backslash P)+|P|-1+\varepsilon(T) .
$$


4.2. Положим $R_{n}(G)=\mathbb{Z} G \otimes K_{n}(G)$. Это - свободный $\mathbb{Z} G$-модуль с базисом, состоящим из элементов вида $1 \otimes C(E) * X(V),|E|+|V|=n$. Покажем, что последовательность

$$
\cdots \stackrel{d}{\longrightarrow} R_{n}(G) \stackrel{d}{\longrightarrow} \cdots \stackrel{d}{\longrightarrow} R_{0}(G) \stackrel{\varepsilon}{\longrightarrow} \mathbb{Z} \longrightarrow 0
$$

является точной ( $(\varepsilon$-аугментация, т.е. $\varepsilon(g \otimes())=1 \forall g \in G)$.

Установим сначала точность в нулевом члене (очевидно, аугментация является эпиморфизмом). Для любого $g \in G$ справедливо следующее:

$$
d\left(g \otimes\left(x_{i}\right)\right)=g\left(x_{i}-1\right) \otimes(), \quad d\left(g \otimes\left(c_{i j}\right)\right)=g\left(c_{i j}-1\right) \otimes() .
$$

Отсюда следует, что $\operatorname{Im}\left\{d: R_{1}(G) \rightarrow R_{0}(G)\right\}=\Delta \otimes($ ) $=\operatorname{Ker}\{\varepsilon\}$ (здесь $\Delta$ обозначает фундаментальный идеал $\mathbb{Z} G)$.

Чтобы доказать точность последовательности (4.1) при $n>0$, рассмотрим следующую конечную возрастаюшую фильтрацию на $R(G)=\mathbb{Z} G \otimes K(G)$. Положим

$$
F_{p} R_{n}(G)=\bigoplus_{\substack{|E|+|V|=n \\|V| \leqslant p}} \mathbb{Z} G \otimes C(E) * X(V) .
$$

Из предложения 3.1 следует, что дифференциал $d$ сохраняет эту фильтрацию. Пусть $\left\{\mathscr{E}_{p q}^{t}, d_{p q}^{t}\right\}$ - спектральная последовательность, ассоциированная с данной фильтрацией и сходящаяся к $H_{p+q} R(G)$.

Шаг 1. Рассмотрим нулевой член этой спектральной последовательности:

$$
\mathscr{E}_{p q}^{0}=F_{p} R_{p+q}(G) / F_{p-1} R_{p+q}(G) \cong \bigoplus_{|E|=q,|V|=p} \mathbb{Z} G \otimes C(E) * X(V),
$$

a $d_{p q}^{0}: \mathscr{E}_{p q}^{0} \rightarrow \mathscr{E}_{p, q-1}^{0}-$ это $G$-гомоморфизм такой, что

$$
d_{p q}^{0}(1 \otimes C(E) * X(V))=\sum_{i=1}^{q}(-1)^{i-1}\left(c\left(e_{i}\right)-1\right) \otimes C\left(E \backslash e_{i}\right) * X(V) .
$$

Для фиксированного $V$ положим

$$
\mathscr{E}_{q}^{0}(V)=\bigoplus_{|E|=q} \mathbb{Z} G \otimes C(E) * X(V)
$$

Ясно, что $\mathscr{E} 0$ является прямой суммой по всем $V$ "вертикальных" комплексов $\mathscr{E}^{0}(V)$ :

$$
\cdots \stackrel{d_{p, q+1}^{0}}{\longrightarrow} \mathscr{E}_{q}^{0}(V) \stackrel{d_{p q}^{0}}{\longrightarrow} \mathscr{E}_{q-1}^{0}(V) \stackrel{d_{p, q-1}^{0}}{\longrightarrow} \cdots, \quad p=|V|,
$$

каждый из которых изоморфен $\mathscr{E}^{0}(\varnothing)$. Исследуем подробно $\mathscr{E}^{0}(\varnothing)$. Пусть $K=$ $Z(G)$ - центр группы $G$ и $\bar{G}=G / K$. Подгруппа $K$ является свободной абелевой группой с базисом $\left\{c(e), e \in \Delta^{1}\right\}$. Хорошо известно, что комплекс свободных левых $\mathbb{Z} K$-модулей

$$
\ldots \stackrel{d_{q+1}(K)}{\longrightarrow} R_{q}(K) \stackrel{d_{q}(K)}{\longrightarrow} R_{q-1}(K) \stackrel{d_{q-1}(K)}{\longrightarrow} \cdots, \quad R_{q}(K)=\bigoplus_{|E|=q} \mathbb{Z} K \otimes C(E),
$$


есть свободная резольвента $\mathbb{Z}$ над $\mathbb{Z} K\left(d_{q}(K)\right.$ - это $K$-гомоморфизм, действующий на элементах $1 \otimes C(E) \in R_{q}(K)$ так же, как и $d_{0 q}^{0}$ на элементах $\left.1 \otimes C(E) \in \mathscr{E}_{q}(\varnothing)\right)$. Нетрудно видеть, что $\mathscr{E} 0(\varnothing) \cong \mathbb{Z} G \otimes_{K} R(K)$ (изоморфизм комплексов левых $\mathbb{Z} G$-модулей $)$, т.е. $\mathscr{E}(\varnothing)$ получается из $R(K)$ индуцированием. Так как $\mathbb{Z} G$ - свободный правый $\mathbb{Z} K$-модуль, то комплекс $\mathscr{E} 0(\varnothing)$ ацикличен в положительных размерностях. Отсюда вытекает, что при $q>0 \quad \mathscr{E}_{p q}^{1} \cong \operatorname{Ker}\left\{d_{p q}^{0}\right\} / \operatorname{Im}\left\{d_{p, q+1}^{0}\right\}=0$. При $q=0$

$$
\mathscr{E}_{p 0}^{1} \cong \bigoplus_{|V|=p} \operatorname{Coker}\left\{d_{p 1}^{0}: \mathscr{E}_{1}^{0}(V) \rightarrow \mathscr{E}_{0}^{0}(V)\right\}
$$

Поскольку

$$
d_{p 1}^{0}(w \otimes c(e) * X(V))=w(c(e)-1) \otimes X(V), \quad w \in \mathbb{Z} G,
$$

то коядро этого гомоморфизма изоморфно $\mathbb{Z} \bar{G} \otimes X(V)$ (ядро естественного эпиморфизма $\mathbb{Z} G \rightarrow \mathbb{Z} \bar{G}$ порождается как левый идеал элементами $\left.c(e)-1, e \in \Delta^{1}\right)$. В результате получаем, что $\mathscr{E}_{p 0}^{1} \cong \bigoplus_{|V|=p} \mathbb{Z} \bar{G} \otimes X(V)$, причем $\mathscr{E}_{p 0}^{1}$ можно считать левьм $\mathbb{Z} \bar{G}$-модулем в силу тривиальности действия $K$.

Шаг 2. Покажем, что $\left(\mathscr{E}_{*, 0}^{1}, d_{*, 0}^{1}\right)$ является свободной резольвентой $\mathbb{Z}$ над $\mathbb{Z} \bar{G}$. Для этого вычислим $d_{p 0}^{1}: \mathscr{E}_{p 0}^{1} \rightarrow \mathscr{E}_{p-1,0}^{1}$. Исходньй дифференциал $d$, индуцируюший $d_{p q}^{1}$, представляется в виде суммы $d_{0}+d_{1}+d_{\geqslant 2}$, где $d_{\geqslant 2}$ уменьшает фильтрационную степень не менее чем на 2 единицы. Из этого можно заключить, что

$$
d_{p 0}^{1}(1 \otimes X(V))=\sum_{j=1}^{p}(-1)^{j-1}\left(\bar{x}\left(v_{j}\right)-1\right) \otimes X\left(V \backslash v_{j}\right)
$$

(здесь $\bar{x}\left(v_{j}\right)$ - это образ $x\left(v_{j}\right)$ при эпиморфизме $G \rightarrow \bar{G}$ ). Поскольку $\bar{G}-$ свободная абелева группа с порождающими $\bar{x}_{j}, j \in \Delta^{0}$, а $d_{p 0}^{1}-\bar{G}$-гомоморфизм по модулю $\operatorname{Im}\left\{d_{p 1}^{0}\right\}$, то комплекс $\left(\mathscr{E}_{*, 0}^{1}, d_{*, 0}^{1}\right)$ действительно оказывается свободной резольвентой $\mathbb{Z}$ над $\mathbb{Z} \bar{G}$. Таким образом, спектральная последовательность $\left\{\mathscr{E}_{p q}^{t}, d_{p q}^{t}\right\}$ вырождается на втором члене и $\mathscr{E}_{p q}^{2}=0$, если $p+q>0$. Отсюда следует ацикличность комплекса (4.1) в положительных размерностях.

Используя явный вид изоморфизма комплексов $(K(G), d) \cong(\Lambda(\mathscr{G}), d)$ из леммы 3.3 , мы получаем изоморфизм резольвент $(R(G), d) \cong(\mathscr{R}(G), d)$ (резольвента $\mathscr{R}(G)$ была определена в п. 1.1). Таким образом, теорема 1 нами полностью доказана.

СлЕДСТвИЕ 4.1. Группы гомологий $H_{*} G$ изоморфны соответствующим группам гомологий комплекса $(\Lambda(\mathscr{G}), d)$.

\section{§5. Изоморфизм $H_{n} G$ и $H_{n} \mathscr{G}$}

В этом параграфе будет дано доказательство теорем 2 и 3.

5.1. Пусть $G=G(r)$ - свободная нильпотентная группа класса 2 с $r$ свободными порождающими, $\mathscr{G}=\mathscr{G}(r)$ - соответствуюшее кольцо Ли. В следствии 4.1 был установлен изоморфизм целочисленных групп гомологий $H_{n} G$ и $n$-х групп гомологий комплекса $(\Lambda(\mathscr{G}), d)$, определенного в конце п. 3.3. Для дальнейшего изложения 
группа $G$ (вернее, ее копредставление) больше не понадобится в явном виде, поэтому мы упростим обозначения, полагая, что $\mathscr{G}$ задается копредставлением

$$
\mathscr{G}=\left\langle x_{i}, c_{i j}, 1 \leqslant i<j \leqslant r \mid c_{i j}=\left[x_{i}, x_{j}\right],\left[c_{i j}, x_{k}\right]=\left[c_{i j}, c_{k l}\right]=0\right\rangle
$$

(черту больше не пишем). Стандартный способ определения групп гомологий $H_{n} \mathscr{G}$ с коэффициентами в тривиальном $\mathscr{G}$-модуле $\mathbb{Z}$ заключается в следуюшем. Рассматривается комплекс $(\Lambda(\mathscr{G}), \partial)$ :

$$
\cdots \stackrel{\partial}{\longrightarrow} \Lambda^{n}(\mathscr{G}) \stackrel{\partial}{\longrightarrow} \cdots \stackrel{\partial}{\longrightarrow} \Lambda^{0}(\mathscr{G}) \longrightarrow 0,
$$

в котором

$$
\partial\left(z_{1} \wedge \cdots \wedge z_{n}\right)=\sum_{1 \leqslant i<j \leqslant n}(-1)^{i+j-1}\left[z_{i}, z_{j}\right] \wedge z_{1} \wedge \cdots \widehat{z}_{i} \cdots \widehat{z}_{j} \cdots \wedge z_{n} .
$$

Как уже отмечалось ранее, базис в $\Lambda^{n}(\mathscr{G})$ составляют элементы вида $C(E) \wedge X(V)$, где $E \subseteq \Delta^{1}, V \subseteq \Delta^{0},|E|+|V|=n$. Действие $\partial$ на базисных элементах определяется, следовательно, двумя свойствами:

1) $\partial C(E) \wedge X(V)=(-1)^{|E|} C(E) \wedge \partial X(V)$;

2) $\partial X(V)=\sum_{L \subseteq V,|L|=2}(-1)^{\varepsilon(L, V \backslash L)} c(L) \wedge X(V \backslash L)$, т.е. $\partial=d_{2}$ в обозначениях п. $3.3, \bar{d}=d_{2}+d_{3}+\cdots+d_{r}$.

Целью этого параграфа является установление изоморфизма между комплексами $(\Lambda(\mathscr{G}), \partial)$ и $(\Lambda(\mathscr{G}), d)$. Достаточно указать автоморфизм $F: \Lambda(\mathscr{G}) \longrightarrow \Lambda(\mathscr{G})$ градуированного $\mathbb{Z}$-модуля $\Lambda(\mathscr{G})$, для которого коммутативна диаграмма

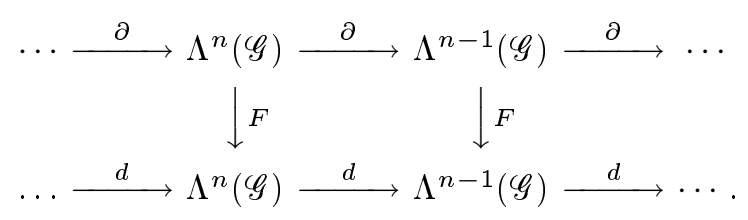

Отметим, что $\Lambda(\mathscr{G})$ является в действительности биградуированной алгеброй:

$$
\Lambda(\mathscr{G})=\bigoplus_{q, p \geqslant 0} \Lambda^{q, p}(\mathscr{G})
$$

где базис $\Lambda^{q, p}(\mathscr{G})$ составляют элементы $C(E) \wedge X(V),|E|=q,|V|=p$. Очевидно, что дифференциал $d_{k}$ из разложения $d=d_{2}+\cdots+d_{r}$ принадлежит группе $\operatorname{Homgr}^{k-1,-k}(\Lambda(\mathscr{G}), \Lambda(\mathscr{G}))$.

Будем искать $F$ в виде $F=1+f_{1}+\cdots+f_{r}$, где 1 означает тождественное отображение, а $f_{k} \in \operatorname{Homgr}^{k,-k}(\Lambda(\mathscr{G}), \Lambda(\mathscr{G}))$, т.е. $f_{k}$ при действии на любой базисньй элемент $\Lambda(\mathscr{G})$ должен уменьшать число свободных порождаюших на $k$ и на столько же увеличивать число коммутаторов. Поскольку $\left(f_{1}+\cdots+f_{r}\right)^{r+1}=0$, любое такое отображение $F$ является автоморфизмом градуированного модуля $\Lambda(\mathscr{G})$.

Чтобы решить уравнение $d F=F \partial$, подставим в него выражения для $d, \partial$ и $F$. Получим

$$
\left(d_{2}+\cdots+d_{r}\right)\left(1+f_{1}+\cdots+f_{r}\right)=\left(1+f_{1}+\cdots+f_{r}\right) d_{2} .
$$


Поскольку все $d_{k}, f_{k}$ являются однородными, то данное уравнение распадается на следуюшую систему:

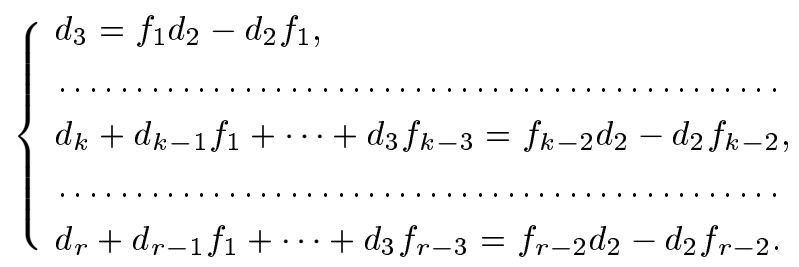

Два уравнения, в которые входят $f_{r-1}, f_{r}$, имеют вид $0=0$, т.е. при фиксированном $r f_{r-1}, f_{r}$ не играют особой роли.

Пусть

$$
\operatorname{Homgr}(\Lambda(\mathscr{G}), \Lambda(\mathscr{G}))=\bigoplus_{q, p} \operatorname{Homgr}^{q, p}(\Lambda(\mathscr{G}), \Lambda(\mathscr{G})) .
$$

Отдельно рассматривая слагаемые, для которых $p+q \equiv 0(\bmod 2)$ или $p+q \equiv 1$ $(\bmod 2)$, получим разложение

$$
\operatorname{Homgr}(\Lambda(\mathscr{G}), \Lambda(\mathscr{G}))=\operatorname{Homgr}_{0}(\Lambda(\mathscr{G}), \Lambda(\mathscr{G})) \oplus \operatorname{Homgr}_{1}(\Lambda(\mathscr{G}), \Lambda(\mathscr{G}))
$$

(т.е. Homgr $(\Lambda(\mathscr{G}), \Lambda(\mathscr{G}))$ является супералгеброй). Как обычно, соответствуюшие эндоморфизмы будем назьвать четныци или нечетными. Ясно, что все $f_{k}$ - четные, а все $d_{k}$ - нечетные эндоморфизмы. Кроме того, на биградуированном $\mathbb{Z}$-модуле $\operatorname{Homgr}(\Lambda(\mathscr{G}), \Lambda(\mathscr{G}))$ зададим дифференциал $D$ полной степени $(-1)$ формулой $D f=d_{2} f-f d_{2}$.

Чтобы получить решение системы (5.1), наложим некоторые дополнительные условия на $f_{1}, \ldots, f_{r}$. Для начала дадим два определения.

ОПРеДЕЛЕнИЕ 5.1. Эндоморфизм $f \in \operatorname{Homgr}(\Lambda(\mathscr{G}), \Lambda(\mathscr{G}))$ назьвается әндоморфизмом типа $(0, q, p)$, если

1) $f \in \operatorname{Homgr}^{p,-p}(\Lambda(\mathscr{G}), \Lambda(\mathscr{G})) \subset \operatorname{Homgr}_{0}(\Lambda(\mathscr{G}), \Lambda(\mathscr{G}))$;

2) $f\left(\Lambda^{s, t}(\mathscr{G})\right)=0$, если $s<q$ или $t<p$;

3) для любых $E, V$

$$
\begin{aligned}
& f(C(E) \wedge X(V)) \\
& =\sum_{\substack{B \subseteq E \\
|B|=q}} \sum_{\substack{R \subseteq V \\
|R|=p}}(-1)^{\mu} C(E \backslash B) \wedge f(C(B) \wedge X(R)) \wedge X(V \backslash R), \\
& \mu=\varepsilon(E \backslash B, B)+\varepsilon(R, V \backslash R) .
\end{aligned}
$$

ОПРеДЕЛЕНИЕ 5.2. Эндоморфизм $f \in \operatorname{Homgr}(\Lambda(\mathscr{G}), \Lambda(\mathscr{G}))$ назьвается әндоморфизмом типа $(1, q, p)$, если

1) $f \in \operatorname{Homgr}^{p-1,-p}(\Lambda(\mathscr{G}), \Lambda(\mathscr{G})) \subset \operatorname{Homgr}_{1}(\Lambda(\mathscr{G}), \Lambda(\mathscr{G}))$;

2) $f\left(\Lambda^{s, t}(\mathscr{G})\right)=0$, если $s<q$ или $t<p$;

3) для любых $E, V$

$$
\begin{aligned}
& f(C(E) \wedge X(V)) \\
& \quad=(-1)^{|E|-q} \sum_{\substack{B \subseteq E \\
|B|=q|R|=p}} \sum_{\substack{R \subseteq V \\
|R|=}}(-1)^{\mu} C(E \backslash B) \wedge f(C(B) \wedge X(R)) \wedge X(V \backslash R),
\end{aligned}
$$

$\mu$ - то же, что и в определении 5.1. 
Другими словами, эндоморфизмы типа $(0, q, p)$ (типа $(1, q, p))$ являются четными (нечетными) и полностью определяются своими значениями на $\Lambda^{q, p}(\mathscr{G})$. В дальнейшем мы будем ссылаться на это свойство как на принцип локализации.

Связь между эндоморфизмами этих двух типов устанавливается в следуюшей лемме.

ЛЕмма 5.1. Если $f$ - эндоморфизм типа $(0, q, p)$, mо $-D f=f d_{2}-d_{2} f$ является әндоморфизмом типа $(1, q-1, p+2)$.

ДокаЗАтЕльство. Ясно, что $D f \in \operatorname{Homgr}^{p+1,-(p+2)}(\Lambda(\mathscr{G}), \Lambda(\mathscr{G})) . \quad$ Если $s<q-1$ или $t<p+2$, то $\left(d_{2} f-f d_{2}\right)\left(\Lambda^{s, t}(\mathscr{G})\right)=0$, что проверяется непосредственно. Покажем, что свойство 3$)$ также выполнено.

С одной стороны,

$$
\begin{aligned}
& d_{2} f(C(E) \wedge X(V)) \\
& \quad=\sum_{\substack{B \subseteq E \\
|B|=q}} \sum_{\substack{P \subseteq V \\
|P|=p}} \sum_{\substack{\subseteq \subseteq V \backslash P \\
|\bar{L}|=2}}(-1)^{\mu_{1}} C(E \backslash B) \wedge c(L) \wedge f(C(B) \wedge X(P)) \wedge X(V \backslash P \backslash L), \\
& \mu_{1}=\varepsilon(E \backslash B, B)+\varepsilon(P, V \backslash P)+\varepsilon(L, V \backslash P \backslash L)+|E|+q .
\end{aligned}
$$

$\mathrm{C}$ другой стороны,

$$
\begin{aligned}
& f d_{2}(C(E) \wedge X(V)) \\
& =\sum_{\substack{L \subseteq V \\
|L|=2}}(-1)^{|E|+\varepsilon(L, V \backslash L)} f(C(E) \wedge c(L) \wedge X(V \backslash L)) \\
& =\sum_{\substack{B \subseteq E \\
|B|=q \mid L \subseteq V}} \sum_{\substack{P \subseteq V \backslash L \\
|L|=2}}(-1)^{\mu_{2}} C(E \backslash B) \wedge c(L) \wedge f(C(B) \wedge X(P)) \wedge X(V \backslash P \backslash L) \\
& +\sum_{\substack{Q \subseteq E \\
|Q|=q}} \sum_{\substack{L \subseteq V \\
|L| L \mid=2}} \sum_{P \subseteq V \backslash L}(-1)^{\lambda} C(E \backslash Q) \wedge f(C(Q) \wedge c(L) \wedge X(P)) \wedge X(V \backslash P \backslash L) .
\end{aligned}
$$

В последнем равенстве

$$
\begin{aligned}
\mu_{2} & =\varepsilon(E \backslash B, B)+\varepsilon(L, V \backslash L)+\varepsilon(P, V \backslash L \backslash P)+|E|+q, \\
\lambda & =\varepsilon(E \backslash Q, Q)+\varepsilon(L, V \backslash L)+\varepsilon(P, V \backslash P \backslash L)+|E| .
\end{aligned}
$$

Подсчет показьвает, что $\mu_{1}$ и $\mu_{2}$ имеют одинаковую четность, следовательно, $D f(C(E) \wedge X(V))$ равняется последней тройной сумме, которая, в свою очередь, равна, как несложно убедиться,

$$
\sum_{\substack{Q \subseteq E \\|Q|=q-1}} \sum_{\substack{R \subseteq V \\|R|=p+2}}(-1)^{\tau+|E|-q} C(E \backslash Q) \wedge f d_{2}(C(Q) \wedge X(R)) \wedge X(V \backslash R),
$$

где $\tau=\varepsilon(E \backslash Q, Q)+\varepsilon(R, V \backslash R), R=P \sqcup L$. По свойству 2) эндоморфизма типа $(0, q, p) d_{2} f(C(Q) \wedge X(R))=0$. Поэтому для $-D f$ действительно выполнено свойство 3$)$ эндоморфизма типа $(1, q-1, p+2)$. Лемма доказана. 
Вернемся к решению системы (5.1). Рассмотрим более простую систему

$$
\left\{\begin{array}{l}
d_{3}=f_{(1)}^{(1)} d_{2}-d_{2} f f_{(1)}^{(1)}=-D f_{(1)}^{(1)} \\
\ldots \ldots \ldots \ldots \ldots \ldots \ldots \ldots \ldots \ldots \ldots \ldots \ldots \ldots \ldots \ldots \ldots \ldots \\
d_{r}=f_{(r-2)}^{(1)} d_{2}-d_{2} f_{(r-2)}^{(1)}=-D f_{(r-2)}^{(1)}
\end{array}\right.
$$

Поскольку $d_{k}$ имеет тип $(1,0, k)$, то $f_{(k-2)}^{(1)}$ надо искать среди эндоморфизмов типа $(0,1, k-2)$. Пусть $|V|=k$. Тогда

$$
d_{k} X(V)=\sum_{T \in \max (V)}(-1)^{\varepsilon(T)} c_{1}(T) \wedge \cdots \wedge c_{k-1}(T)=\sum_{T \in \max (V)}(-1)^{\varepsilon(T)} C(T)
$$

С другой стороны, $-D f_{(k-2)}^{(1)}(X(V))=f_{(k-2)}^{(1)} d_{2} X(V)$, так как $f_{(k-2)}^{(1)}\left(\Lambda^{0, k}(\mathscr{G})\right)=0$. Поэтому достаточно (по принципу локализации), чтобы всегда было выполнено равенство

$$
\begin{aligned}
f_{(k-2)}^{(1)} d_{2} X(V) & =\sum_{L \subseteq V,|L|=2}(-1)^{\varepsilon(P, V \backslash L)} f_{(k-2)}^{(1)}(c(L) \wedge X(V \backslash L)) \\
& =\sum_{T \in \max (V)}(-1)^{\varepsilon(T)} C(T) .
\end{aligned}
$$

Элементы $c_{i j} \wedge X(P),|P|=k-2$, составляют базис $\Lambda^{1, k-2}(\mathscr{G})$. Определим $f_{(k-2)}^{(1)}$ на элементах базиса. Если $\left\{v_{i}, v_{j}\right\} \cap P \neq \varnothing$, то $f_{(k-2)}^{(1)}\left(c_{i j} \wedge X(P)\right)=0$. Для остальных базисных элементов (а они имеют вид $c(L) \wedge X(V \backslash L)$, где $L=$ $\left.\left\{v_{i}, v_{j}\right\}, V=P \sqcup L\right)$ значение $f_{(k-2)}^{(1)}(c(L) \wedge X(V \backslash L)) \in \Lambda^{k-1,0}(\mathscr{G})$ может быть произвольным, но при этом должно выполняться единственное соотношение (для фиксированного $V$ ):

$$
\sum_{L \subseteq V,|L|=2}(-1)^{\varepsilon(L, V \backslash L)} f_{(k-2)}^{(1)}(c(L) \wedge X(V \backslash L))=\sum_{T \in \max (V)}(-1)^{\varepsilon(T)} C(T),
$$

т.е. имеется достаточно много степеней свободы в определении $f_{(k)}^{(1)}$.

5.2. Решение системы (5.1). Зафииксируем какое-либо решение системы (5.2), т.е. набор эндоморфизмов $\left(f_{(1)}^{(1)}, \ldots, f_{(r-2)}^{(1)}\right)$, для которых $-D f_{(k)}^{(1)}=d_{k+2}$. Оказывается, что эндоморфизмы $f_{p}$, удовлетворяющие системе (5.1), можно получить в следующем виде:

$$
f_{p}=\sum_{\substack{t_{1}>\cdots>t_{n} \geqslant 1 \\ q_{1} t_{1}+\cdots+q_{n} t_{n}=p, q_{i} \geqslant 1}} f_{\left(t_{1}, \ldots, t_{n}\right)}^{\left(q_{1}, \ldots, q_{n}\right)}, \quad p=1, \ldots, r-2 .
$$

Эндоморфизмы $f_{\left(t_{1}, \ldots, t_{n}\right)}^{\left(q_{1}, \ldots, q_{n}\right)}$ имеют тип $(0, q, p)$, где $q=\sum_{1 \leqslant i \leqslant n} q_{i}$, и определяются индуктивно по принципу локализации $(|E|=q-1,|V|=p)$ :

$$
\begin{aligned}
& f_{\left(t_{1}, \ldots, t_{n}\right)}^{\left(q_{1}, \ldots, q_{n}\right)}(C(E) \wedge c \wedge X(V)) \\
& \quad=\sum_{1 \leqslant i \leqslant n} \sum_{\substack{R \subset V \\
|V \backslash R|=t_{i}}}(-1)^{\nu_{i}} f_{\left(t_{1}, \ldots, t_{i}, \ldots, t_{n}\right)}^{\left(q_{1}, \ldots, q_{i-1}, \ldots, q_{n}\right)}(C(E) \wedge X(R)) \wedge f_{t_{i}}^{1}(c \wedge X(V \backslash R)),
\end{aligned}
$$


$\nu_{i}=p-t_{i}+\varepsilon(R, V \backslash R)$. При этом если некоторое $q_{i}=1$, то в правой части вычеркивается как $q_{i}-1=0$, так и нижнее $t_{i}$.

Отметим, что слагаемые, из которых состоит $f_{p}$, параметризуются всеми диаграммами Юнга, состоящими из $p$ клеток (имеющими $q_{1}$ строк длины $t_{1}, \ldots, q_{n}$ строк длины $\left.t_{n}\right)$.

Выделение именно последнего коммутатора при индуктивном определении эндоморфизмов $f=f_{\left(t_{1}, \ldots, t_{n}\right)}^{\left(q_{1}, \ldots, q_{n}\right)}$ носит весьма условньй характер (аналогично разложению определителя по последней строке). На самом деле $f$ обладает следующим свойством:

$$
f\left(c_{1} \wedge \cdots \wedge c_{q} \wedge X(V)\right)=(-1)^{\widetilde{\pi}} f\left(c_{\pi(1)} \wedge \cdots \wedge c_{\pi(q)} \wedge X(V)\right)
$$

где $\pi$ - перестановка из $S_{q}$. По индукции и принципу локализации можно свести проверку к случаю $q=2$. Пусть $f=f_{(s, t)}^{(1,1)}, s>t$, или $f=f_{(s)}^{(2)}$. Рассмотрим первый случай. Тогда

$$
\begin{aligned}
f_{(s, t)}^{(1,1)}\left(c_{1} \wedge c_{2} \wedge X(V)\right)= & \sum_{\substack{R \subset V \\
|R|=s}}\left((-1)^{t+\varepsilon(R, V \backslash R)} f_{s}^{1}\left(c_{1} \wedge X(R)\right) \wedge f_{t}^{1}\left(c_{2} \wedge X(V \backslash R)\right)\right. \\
& \left.\quad+(-1)^{s+\varepsilon(V \backslash R, R)} f_{t}^{1}\left(c_{1} \wedge X(V \backslash R)\right) \wedge f_{t}^{1}\left(c_{2} \wedge X(R)\right)\right) .
\end{aligned}
$$

С другой стороны,

$$
\begin{aligned}
f_{(s, t)}^{(1,1)}\left(c_{2} \wedge c_{1} \wedge X(V)\right)= & \sum_{\substack{R \subset V \\
|R|=s}}\left((-1)^{t+\varepsilon(R, V \backslash R)} f_{s}^{1}\left(c_{2} \wedge X(R)\right) \wedge f_{t}^{1}\left(c_{1} \wedge X(V \backslash R)\right)\right. \\
& \left.\quad+(-1)^{s+\varepsilon(V \backslash R, R)} f_{t}^{1}\left(c_{2} \wedge X(V \backslash R)\right) \wedge f_{t}^{1}\left(c_{1} \wedge X(R)\right)\right) .
\end{aligned}
$$

При этом

$$
\varepsilon(V \backslash R, R)+s+(1+s)(1+t) \equiv \varepsilon(R, V \backslash R)+t+1 \quad(\bmod 2)
$$

т.е. вьшеуказанные суммы отличаются только знаком.

Аналогично,

$$
\begin{aligned}
f_{(s)}^{(2)}\left(c_{1} \wedge c_{2} \wedge X(V)\right) & =\sum_{\substack{R \subset V \\
|R|=s}}(-1)^{\varepsilon(R, V \backslash R)+s} f_{s}^{1}\left(c_{1} \wedge X(R)\right) \wedge f_{s}^{1}\left(c_{2} \wedge X(V \backslash R)\right) \\
& =-f_{(s)}^{(2)}\left(c_{2} \wedge c_{1} \wedge X(V)\right) .
\end{aligned}
$$

Лемма 5.2. В $\operatorname{Homgr}(\Lambda(\mathscr{G}), \Lambda(\mathscr{G}))$ имеет место равенство

$$
-D f_{\left(t_{1}, \ldots, t_{n}\right)}^{\left(q_{1}, \ldots, q_{n}\right)}=\sum_{i=1}^{n} d_{t_{i}+2} f_{\left(t_{1}, \ldots, t_{i}, \ldots, t_{n}\right)}^{\left(q_{1}, \ldots, q_{i}-1, \ldots, q_{n}\right)}
$$


ДокАЗАТЕЛЬСТво. Несложно показать, используя схему доказательства леммы 5.1 , что $d_{k} f$ является эндоморфизмом типа $(1, q, p+k)$, если $f$ имеет тип $(0, q, p)$. Поэтому как левая, так и правая часть доказываемого равенства имеют тип $(1, q-1, p+2)$. В соответствии с принципом локализации достаточно проверить равенство этих двух эндоморфизмов, ограничив их на $\Lambda^{q-1, p+2}(\mathscr{G})$.

Пусть $C(E) \wedge X(V)$ - базисный элемент в $\Lambda^{q-1, p+2}(\mathscr{G})$. Обозначим через $f$ эндоморфизм $f_{\left(t_{1}, \ldots, t_{n}\right)}^{\left(q_{1}, \ldots, q_{n}\right)}$, через $f^{(i)}-$ эндоморфизм $f_{\left(t_{1}, \ldots, t_{i}, \ldots, t_{n}\right)}^{\left(q_{1}, \ldots, q_{n}\right)}$. С одной стороны,

$$
\begin{aligned}
- & D f(C(E) \wedge X(V))=f d_{2}(C(E) \wedge X(V)) \\
= & \sum_{\substack{L \subset V \\
|L|=2}}(-1)^{|E|+\varepsilon(L, V \backslash L)} f(C(E) \wedge c(L) \wedge X(V \backslash L)) \\
= & \sum_{i=1}^{n} \sum_{\substack{L \subset V \\
|L|=2}} \sum_{\substack{R \subset V \backslash L\left|=p-t_{i}\\
\right| R \mid}}(-1)^{\lambda_{i}} f^{(i)}(C(E) \wedge X(R)) \wedge f_{\left(t_{i}\right)}^{(1)}(c(L) \wedge X(V \backslash R \backslash L)) \\
= & \sum_{i=1}^{n} \sum_{\substack{R \subset V \\
|R|=p-t_{i}}}(-1)^{\mu_{i}} f^{(i)}(C(E) \wedge X(R)) \wedge f_{\left(t_{i}\right)}^{(1)} d_{2} X(V \backslash R) \\
= & \sum_{i=1}^{n} \sum_{\substack{R \subset V \\
|R|=p-t_{i}}}(-1)^{\mu_{i}} f^{(i)}(C(E) \wedge X(R)) \wedge d_{t_{i}+2} X(V \backslash R),
\end{aligned}
$$

где $\lambda_{i}=|E|+p-t_{i}+\varepsilon(L, V \backslash L)+\varepsilon(R, V \backslash L \backslash R), \mu_{i}=|E|+p-t_{i}+\varepsilon(R, V \backslash R)$.

С другой стороны,

$$
\begin{aligned}
& \sum_{i=1}^{n} d_{t_{i}+2} f^{(i)}(C(E) \wedge X(V)) \\
& \quad=\sum_{i=1}^{n} d_{t_{i}+2} \sum_{\substack{R \subset V \\
|R|=p-t_{i}}}(-1)^{\varepsilon_{i}} f^{(i)}(C(E) \wedge X(R)) \wedge X(V \backslash R) \\
& \quad=\sum_{i=1}^{n} \sum_{\substack{R \subset V \\
|R|=p-t_{i}}}(-1)^{\mu_{i}} f^{(i)}(C(E) \wedge X(R)) \wedge d_{t_{i}+2} X(V \backslash R),
\end{aligned}
$$

где $\varepsilon_{i}=\varepsilon(R, V \backslash R)$, а $\mu_{i}-$ то же, что и выше. Лемма доказана.

Если подставить выражение для $f_{p}$ в правую часть $p$-го уравнения системы (5.1), воспользоваться леммой 5.2 и собрать в левой части все слагаемые с фиксированньм множителем $d_{k}, k=3, \ldots, p+2$, то получится как раз равенство

$$
-D f_{p}=d_{p+2}+d_{p+1} f_{1}+\cdots+d_{3} f_{p-1} .
$$

Таким образом, теорема 2, а вместе с ней и теорема 3 доказаны. 


\section{§6. Доказательство теоремы 4}

Предположим, что в расширении

$$
1 \longrightarrow A \longrightarrow G \longrightarrow Q \longrightarrow 1
$$

группа $A$ - свободная абелева. Тогда, как известно [10], [11], дифференциалы $d_{p q}^{2}$ спектральной последовательности расширения (6.1) можно представить в виде $d_{p q}^{2}=f_{p q}+\delta_{p q}^{2}$, где

$$
f_{p q}: H_{p}\left(Q, \Lambda^{q}(A)\right) \rightarrow H_{p-2}\left(Q, \Lambda^{q+1}(A)\right)
$$

- умножение на 2-коцикл, задающий расширение (6.1), а $\delta_{p q}^{2}$ - дифференциал спектральной последовательности расшепляюшегося расширения $Q$ с помошью $A$, в котором $Q$ действует на $A$ так же, как в (6.1). Если расширение (6.1) к тому же центрально, то, очевидно, второе слагаемое тривиально и $d_{p q}^{2}=f_{p q}$. Пусть $B(Q)$ - стандартная резольвента группы $Q$. Тогда $H_{p}\left(Q, \Lambda^{q}(A)\right)$ вычисляется с помощью комплекса

$$
\Lambda^{q}(A) \otimes_{Q} B(Q) .
$$

Если $f: Q \times Q \rightarrow A-2$-коцикл, задаюший расширение, то, как известно, умножение на $f$, т.е. гомоморфизм $f_{p q}$, индуцируется отображением

$$
a_{1} \wedge \cdots \wedge a_{q} \otimes\left(y_{1}, \ldots, y_{p}\right) \rightarrow a_{1} \wedge \cdots \wedge a_{q} \wedge f\left(y_{1}, y_{2}\right) \otimes\left(y_{3}, \ldots, y_{p}\right) .
$$

Применим это к случаю, когда $G$ - свободная нильпотентная группа класса 2 . В качестве системы представителей группы $G / G^{\prime}$ можно взять инверсные произведения $x_{k_{1}}^{m_{1}} \cdots x_{k_{n}}^{m_{n}} \in G, k_{1}>\cdots>k_{n}$. Тогда для соответствуюшей системы факторов

$$
f\left(x_{i}, x_{j}\right)=0 \text { при } i>j, \quad f\left(x_{i}, x_{j}\right)=\left[x_{i}, x_{j}\right] \text { при } i<j .
$$

В комплексе (6.2) элементы группы $E_{p q}^{2}=\Lambda^{q}\left(G^{\prime}\right) \otimes \Lambda^{p}\left(G / G^{\prime}\right)$ представляются циклами

$$
c_{i_{1} j_{1}} \wedge \cdots \wedge c_{i_{q} j_{q}} \otimes \sum_{\sigma \in S_{p}}(-1)^{\operatorname{sign}(\sigma)}\left(x_{\sigma\left(k_{1}\right)}, \ldots, x_{\sigma\left(k_{q}\right)}\right)
$$

(суммирование происходит по всем подстановкам на множестве из $p$ элементов). Учитывая (6.4), применим к этим элементам отображение (6.3). В результате получим

$$
\begin{aligned}
\sum_{i<j}(-1)^{i+j-1} c_{i_{1} j_{1}} \wedge \cdots \wedge & c_{i_{q} j_{q}} \wedge\left[x_{k_{i}}, x_{k_{j}}\right] \\
& \otimes \sum_{\sigma \in S_{p-2}}(-1)^{\operatorname{sign}(\sigma)}\left(x_{k_{1}}, \ldots, \widehat{x}_{k_{i}}, \ldots, \widehat{x}_{k_{j}}, \ldots, x_{k_{p}}\right) .
\end{aligned}
$$

Мы видим, что $f_{p q}$ действует на указанные циклы точно так же, как действует на соответствуюшие циклы дифференциал $\partial$ комплекса $\Lambda(\mathscr{G})$.

Аналогично доказывается, что в спектральной последовательности, построенной для кольца Ли $\mathscr{G}, d_{p q}^{2}$ тоже вычисляется как дифференциал комплекса $\Lambda(\mathscr{G})$. 
Но гомологии этого комплекса изоморфны группам $H_{n} \mathscr{G}$, следовательно, на членах $E_{p q}^{3}$ должна происходить стабилизация. Более того, так как

$$
\Lambda^{n}(\mathscr{G})=\bigoplus_{p+q=n} \Lambda^{q}\left(\mathscr{G}^{\prime}\right) \otimes \Lambda^{p}\left(\mathscr{G} / \mathscr{G}^{\prime}\right)
$$

то $H_{n} \mathscr{G}$ распадается в прямую сумму

$$
H_{n} \mathscr{G}=\bigoplus_{p+q=n} E_{p q}^{3}
$$

Теперь вспомним, что для групшы $G$ члены $E_{p q}^{2}$ и дифференциалы $d_{p q}^{2}$ устроены так же, как для кольца $\mathscr{G}$, поэтому одинаковы и члены $E_{p q}^{3}$. По теореме 3 имеет место изоморфизм $H_{n} G \cong H_{n} \mathscr{G}$, откуда следует, что в спектральной последовательности группы $G$ на членах $E_{p q}^{3}$ происходит стабилизация, а соответствуюшая фильтрация расшепляется.

\section{Список литературы}

1. Kuzmin Yu. V. Homology theory of free abelianized extensions // Comm. Algebra. 1988. V. 16. P. $2447-2533$.

2. Кузьмин Ю. В. О некоторых свойствах свободных абелевых расширений // Матем. сб. 1989. T. 180. №6. C. 850-862.

3. Ковач Л. Г., Кузьмин Ю. В., Штер Р. Гомология свободншх абелевых расширений // Матем. сб. 1991. Т. 182. № 4. С. 526-542.

4. Кузьмин Ю. В. О гомологиях свободной разрешимой группы // Матем. заметки. 1993. T. 54. №6. C. 56-65.

5. Кузьмин Ю. В. О связи между когомологиями групп и алгебр Ли // УМН. 1982. Т. 37. № 4. C. 161-162.

6. Lambe L. A., Priddy S. B. Cohomology of nilmanifolds and torsion free nilpotent groups // Trans. Amer. Math. Soc. 1982. V. 273. P. 39-55.

7. Huebschmann J. Perturbation theory and free resolutions for nilpotent groups of class 2 // J. Algebra. 1989. V. 126. № 2. P. 348-399.

8. Lambe L. A. Cohomology of principal G-bundles over a torus when $H^{*}(B G ; R)$ is polynomial // Bull. Soc. Math. Belg. Ser. A. 1986. V. 38. P. 247-264.

9. Sigg St. Laplacian and homology of free 2-step nilpotent Lie algebras // J.Algebra. 1996. V. 185. №1. P. 144-161.

10. André $M$. Le $d_{2}$ de la suite spectrale en cohomologie des groupes // C. R. Acad. Sci. Paris. Sér. I Math. 1965. V. 260. P. 2669-2671.

11. Кузьмин Ю. В. О дифференциалах спектральной последовательности группового расширения // Матем. сб. 1987. Т. 133. №1. С. 49-63. 